\title{
Voltage-Gated Potassium Channel Kv1.3 as a Therapeutic Target for Pancreatic Ductal Adenocarcinoma
}

\author{
Weiwei Li $^{1}$, Gregory C. Wilson ${ }^{1}$, Magdalena Bachmann ${ }^{1,2}$, Jiang Wang ${ }^{3}$, Andrea Mattarei ${ }^{4}$, \\ Cristina Paradisi ${ }^{5}$, Michael J. Edwards ${ }^{1}$, Ildiko Szabo ${ }^{2,6}$, Erich Gulbins ${ }^{1,7}$, Syed A. Ahmad ${ }^{1}$, and \\ Sameer H. Patel ${ }^{1}$
}

\section{Affiliation List:}

1. Department of Surgery, University of Cincinnati, 231 Albert Sabin Way, Cincinnati, OH 45267-0558, USA

2. Department of Biology, University of Padova, viale G. Colombo 3, 35121 Padova, Italy

3. Department of Pathology, University of Cincinnati, 231 Albert Sabin Way, Cincinnati, OH 45267-0558, USA

4. Department of Pharmaceutical and Pharmacological Sciences, University of Padua, Italy

5. Department of Chemical Sciences, University of Padua, Italy

6. CNR Institute of Neurosciences, University of Padova, viale G. Colombo 3, 35121 Padova, Italy

7. Department of Molecular Biology, University of Duisburg-Essen, Hufelandstrasse 55, 45122 Essen, Germany

\section{Corresponding Author:}

Sameer H. Patel, MD, CMQ, FACS, FSSO

Assistant Professor

Director of Research in Surgical Oncology

Division of Surgical Oncology

University of Cincinnati College of Medicine

231 Albert Sabin Way ML-0558

Cincinnati, $\mathrm{OH} 45267-0558$

Tel: 513-558-6018

Fax: 513-584-0459

Email: patel5se@ucmail.uc.edu

Grant Support: This project was supported by The Central Surgical Association Turcotte Award.

Conflict of Interest Statements: None of the authors have any conflicts of interest to disclose

Presentation: This project was accepted for a poster presentation at the AACR Annual Virtual Meeting June 2020 (E-poster due to COVID-19). 


\begin{abstract}
The mitochondrial voltage-gated potassium channel, Kv1.3, has been emerged as an attractive oncologic target but its function in pancreas cancer (PDAC) is unknown. In this study we evaluated tissue expression of Kv1.3 in resected PDAC from 55 patients and tumor inhibition in orthotopic mouse models using the recently developed Kv1.3 inhibitors PCARBTP and PAPTP. Immunohistochemistry of 55 human PDAC specimens showed that all tumors expressed Kv1.3 with $60 \%$ of tumor specimens having high Kv1.3 expression. In pancreas tumor models (Pan02 cells injected into C57BL/6 mice), PCARBTP and PAPTP treatment resulted in tumor reductions of $87 \%$ and $70 \%$, respectively. When combined with gemcitabine/abraxane, this increased to $95 \%$ and $80 \%$ without resultant organ toxicity. In vivo models indicated PCARBTP-mediated cell death occurred through the p38-MAPK pathway. In vitro-generated resistant clones to PCARBTP escaped cell death through upregulation of the anti-oxidant system as determined using SWATH-MS analysis. These data show Kv1.3 is highly expressed in resected human PDAC and the use of novel mitochondrial Kv1.3 inhibitors combined with cytotoxic chemotherapies might be novel, effective treatment for PDAC.
\end{abstract}

Key Words: pancreas adenocarcinoma, mitochondria, potassium channel, Kv1.3 


\section{Introduction}

Despite the utilization of more aggressive systemic chemotherapy regimens, pancreas ductal adenocarcinoma (PDAC) remains a devastating disease and is the third leading cause of cancer related mortality. ${ }^{1}$ In 2020, pancreas cancer will affect approximately 57,600 patients in the US and the incidence is expected to rise. ${ }^{2}$ Surgery remains the only option for cure but unfortunately, only $15-20 \%$ of patients are candidates for resection and five year overall survival remains less than $20 \%$ with surgery alone. Apart from surgery and chemotherapy, few effective treatment options exist. Immunotherapy, which has shown dramatic results in many gastrointestinal and cutaneous malignancies, thus far has shown minimal benefit in PDAC. One of the barriers to treatment is the tumor microenvironment, which is rich in immunosuppressive cells. ${ }^{3}$ These regulatory $\mathrm{T}$ cells, myeloid derived suppressor cells, and tumor associated macrophages (TAMs) allow tumor cells to evade identification and are involved in the development of resistance. ${ }^{3,4}$ Novel treatment strategies are desperately needed.

Voltage-dependent $\mathrm{K}^{+}$channels (Kv) are a superfamily of ubiquitously expressed membrane proteins that are involved in maintaining membrane resting/action potentials, cell proliferation, immune activation, and cell death. ${ }^{5} \mathrm{Kv} 1.3$ is a specific voltage-dependent $\mathrm{K}^{+}$channel located mainly in the plasma and inner mitochondrial membranes (mitoKv1.3). First discovered in the plasma membrane of human T lymphocytes, Kv1.3 is also found in tumor and immune cells where they regulate proliferation as well as apoptosis and are aberrantly expressed in malignancies. $^{6-8}$ Kv1.3 is inhibited by many chemically unrelated compounds such as smallmolecule organic compounds, and venom-isolated oligopeptides. ${ }^{9-15}$ Our group has recently developed two specific mitoKv1.3 inhibitors that prevalently and specifically target the mitochondrial channel by virtue of a positively charged triphenylphosphonium group. These inhibitors (PCARBTP and PAPTP) were shown to selectively kill cancer cells but not normal healthy cells through a reactive oxygen species (ROS)-mediated cell death involving the respiratory chain complex I. ${ }^{16-18}$ 
In vitro and in vivo treatment of metastatic human pancreas cancer cell lines with mitochondrial Kv1.3 inhibitors resulted in cell death and reduced tumor growth but not complete eradication. ${ }^{16}$ Furthermore, the clinical impact of Kv1.3 in resected human PDAC specimens is not known. Therefore, in this study we sought to evaluate tissue expression of Kv1.3 in resected PDAC, and assess tumor growth inhibition using novel inhibitors of mitoKv1.3 in conjunction with cytotoxic chemotherapy.

\section{Results}

\section{Kv1.3 is Highly Expressed in Resected Human Specimens and PDAC cell lines.}

Immunohistochemical (IHC) evaluation was performed on tissues from 55 patients who were diagnosed at a relatively early stage, in time to undergo resection for PDAC (Figure 1A). Median patient age was 68 years old with $42 \%$ of patients being female and $86 \%$ being white followed by $9 \%$ black. A whipple operation (pancreaticoduodenectomy) was performed in $69.1 \%$ of patients, distal pancreatectomy in $29.1 \%$, and total pancreatectomy in $1.8 \%$. On final pathology, $29.1 \%$ of specimens had poorly differentiated tumors, $90.9 \%$ had perineural invasion, and $56.4 \%$ lymphovascular invasion. Positive lymph nodes were found in $83.6 \%$ of patients. American Joint Committee on Cancer (AJCC $8^{\text {th }}$ edition) staging showed $52.7 \%$ were stage 2 , $34.5 \%$ stage 3 , and $12.7 \%$ were stage 1 .

Kv1.3 IHC staining showed that $96.4 \%(n=53)$ of specimens exhibited expression. Based on percentage and intensity of stain, we found that $60.0 \%$ of tumor specimens had high expression. Only $8.3 \%$ of normal pancreas specimens had expression of Kv1.3. Over a median follow up of 28.0 months, $67.9 \%$ of patients developed a recurrence and $65.5 \%$ of patients died. When stratified by Kv1.3 expression, median recurrence free survival was 16 months with high Kv1.3 expression versus 17 months with low expression $(p=0.36)$ (Figure 1B). Median overall survival was 27 months with high Kv1.3 expression and not reached 30 months for low Kv1.3 expression ( $p=0.53$ ) (Figure 1C). High expression of Kv1.3 in tumor specimens was not 
associated with having a positive lymph node, poor differentiation, perineural invasion, or lymphovascular invasion ( $p>0.05)$.

\section{PCARBTP and PAPTP Reduced Pancreatic Ductal Adenocarcinoma Tumor Size in an Orthotopic} Mouse Model.

Since no studies have been performed on PDAC in immune-competent mice with mitoKv1.3 inhibitors, tumor growth was examined here in the setting of treatment with specific mitoKv1.3 inhibitors in an orthotopic model injecting mouse Pan02 cells. Six days post tumor injection, 1003D mini-pumps (MICRO-OSMOTIC PUMP MODEL 1003D, Reservoir Volume, $100 \mu \mathrm{l}, 1.0 \mu \mathrm{l}$ per hour, 3 days) were implanted filled with $100 \mu \mathrm{L}$ of 50\% DMSO, $25 \mathrm{mM}$ PCARBTP and $50 \mathrm{mM}$ PCARBTP respectively at a flow rate of 1 or $2 \mathrm{nmol} / \mathrm{h} / \mathrm{gbw}$ (Table 1$) .3$ days after implantation, mini-pumps were replaced once fulfilled with same concentration drug at D10, tissues were collected at D14. The tumor volume and mass treated with lower dose of PCARBTP were reduced by $60 \%(n=7)$ and $50 \%(n=7)$, the tumor volume and mass treated with the higher dose PCARBTP were reduced by $72 \%(n=7)$ and $68 \%(n=7)$, respectively (Figure $2 A)$. There were no significant changes in body weights of the mice with drug treatment from the pre-orthotopic injection body weights ( $p=0.84$ for $1 \mathrm{nmol} / \mathrm{h} / \mathrm{gbw}, \mathrm{p}=0.22$ for $2 \mathrm{nmol} / \mathrm{h} / \mathrm{gbw}$, Figure 2B). Representative tumor images are shown in Figure 2C.

An additional drug application method was used with intraperitoneal injection of PCARBTP and PAPTP beginning at 6 days post the tumor injection, and tumors were collected at day 12 post tumor injection. $15 \mathrm{nmol} / \mathrm{g}$ body weight and $20 \mathrm{nmol} / \mathrm{g}$ body weight of PCARBTP were injected every other day for 3 doses (Table 2). Likewise, $5 \mathrm{nmol} / \mathrm{g}$ body weight PAPTP was injected every other day for 3 doses (Table 3). The tumor volume and mass treated with $15 \mathrm{nmol} / \mathrm{gbw}$ PCARBTP were reduced by $87 \%$ and $87 \%(n=8)$. The tumor volume and mass treated with 20 $\mathrm{nmol} / \mathrm{gbw}$ PCARBTP were reduced by $88 \%$ and $90 \%(\mathrm{n}=8)$ (Figure $3 \mathrm{~A}$ ). Although there were decreases in body weight from the pre-orthotopic weight after PCARBTP treatment at both the 15 and $20 \mathrm{nmol} / \mathrm{gbw}$ doses ( $\mathrm{p}=0.02$ and $\mathrm{p}=0.006$, respectively), no mice had greater than a $20 \%$ decrease body weight (Figure 3B). Representative images are shown in Figure 3C. The tumor 
volume and mass treated with $5 \mathrm{nmol} / \mathrm{gbw}$ PAPTP were reduced by $70 \%(\mathrm{n}=8)$ and $64 \%(\mathrm{n}=8)$, respectively (Figure 4A). There were no significant changes in body weights of the mice with PAPTP treatment from the pre-orthotopic injection body weights as shown in Figure 4B $(p=0.17)$. Representative tumors are shown in Figure 4C.

\section{Concomitant Gemcitabine-Abraxane Treatment with PCARBTP/PAPTP Further Reduced Tumor Growth in an Orthotopic Mouse Model.}

We then examined if there was a synergistic effect of using mitoKv1.3 inhibitors with cytotoxic chemotherapies already used in the clinical practice for pancreas cancer. Compared to untreated controls, the tumor volume and mass reduction seen with $190 \mathrm{nmol} / \mathrm{gbw}$ gemcitabine plus $23.4 \mathrm{nmol} / \mathrm{gbw}$ Abraxane (albumin-bound paclitaxel) were only 64\% and 60\% $(n=8)$. However, the tumor volume and mass reduction with $190 \mathrm{nmol} / \mathrm{gbw}$ gemcitabine with $23.4 \mathrm{nmol} / \mathrm{gbw}$ abraxane plus $15 \mathrm{nmol} / \mathrm{gbw}$ PCARBTP were 95\% and 92\%, respectively $(\mathrm{n}=8)$. Similarly, we found substantial tumor volume and mass reduction after treatment with 190 nmol/gbw gemcitabine with $23.4 \mathrm{nmol} / \mathrm{gbw}$ Abraxane and $5 \mathrm{nmol} / \mathrm{gbw}$ PAPTP of $80 \%$ and $75 \%$ (Figure 5).

\section{Combined Treatment with Mitochondrial Kv1.3 Inhibitors and Cytotoxic Chemotherapies had No End Organ Toxicities.}

We have previously reported that PAPTP at $5 \mathrm{nmol} / \mathrm{gbw}$ and PCARBTP at $10 \mathrm{nmol} / \mathrm{gbw}$ were not toxic and did not cause changes in the electrocardiogram of mice ${ }^{16}$. Here we observed that mice did not show sign of distress and show that H\&E staining at the time of sacrifice after treatment with the above described combination of PCARBTP/PAPTP with Gemcitabine/Abraxane (see concentrations above) showed there was no significant toxicity to the heart, lung, liver, and kidney (Figure 6). 


\section{Mitochondrial Kv1.3 Inhibitor PCARBTP Treatment Activated the Phosphorylation of p38 MAPK, SAPK/JNK.}

We have previously shown that the action of sub-lethal concentrations of PCARBTP was exacerbated when the stress response kinase JNK was inhibited, at least in Jurkat lymphocytes 19. Here, we evaluated the effect of various concentrations of PCARBTP on JNK activation monitored by phosphorylation of the kinase, as well as activation of p38 mitogen-activated protein kinase (MAPK), given that both kinases regulate apoptosis induced by several forms of cellular insults. Pan02 cells were treated by PCARBTP (up to $10 \mu \mathrm{M}$ ). Western blot analysis with P-p38 MAPK, P-SAPK/JNK showed there was an increase in phosphorylated levels of these proteins when treating the cells with high concentration of the drugs (Figure 7A).

To test the significance of p38 MAPK for mitoKv1.3 inhibitor mediated cell death, we examined the effects of treatment with the p38 MAPK inhibitor, SB203580. Treatment with $13 \mathrm{nmol} / \mathrm{gbw}$ SB203580 was found to attenuate the effects of PCARBTP in an orthotopic mouse model (Figure 7B). Compared to the vehicle control, tumor volume from all drug treated groups were significantly decreased $(p<0.05)$. SB203580 treatment alone reduced tumor volume by $42 \%$. Compared to PCARBTP only, the addition of SB203580 to PCARBTP decreased growth inhibition by mitoKv1.3 inhibitor treatment (tumor volume $87 \%$ vs $51 \%, p<0.01$ ). Similarly, the addition of SB203580 to Gemcitabine-Abraxane-PCARBTP attenuated the tumor-reducing effect of the drugs, as tumor volume was reduced only by $80 \%$ (versus 95\%) in the presence of p38 MAPK inhibitor $(p<0.05)$, suggesting that PCARBTP mediated cell death occurs at least in part through the p38 MAPK pathway, even in vivo.

\section{Resistance to PCARBTP Therapy}

To examine if resistance to the most efficient mitoKv1.3 inhibitor treatment occurs, we generated a PCARBTP resistant clone using the mouse PDAC cell line we used for the in vivo studies (Pan02), and compared protein expression to normal Pan02 cells by SWATH nanoLCMS/MS. The analysis showed that 50 proteins were upregulated and 8 proteins were downregulated. The upregulated proteins were Fasn, Aldh2, Phgdh, Anxa3, Ldha, Aldoa, Ugdh, PRDX6, Hsph1, Pgk1, Tpi1, Gpi1, Krt19, Esd, Gsto1, Psmd2, Aldh3a1, Acly, Ezr, Arf5, Ephx1, Por, 
G6pdx, LEG3, FRIL1, Serpinb6a, Krt7, Xdh, Vat1, Naca, Gsr, Ywhag, Tfrc, Mif, Pgm1, Akr1c13, Aacs, Gsta2, Plin3, Psph, Hnrnpa1, Ddx39, Pygb, Hars, Eif5, Ranbp3, Hic2, Slc2a1, Uso1, Gng7

(Figure $8 \mathrm{~A}$ and Table I). As indicated by STRING and pathway enrichment analysis, these proteins grouped into a few main functional classes, linked to metabolism of xenobiotics by cytochrome P450 (Figure 8B), the anti-oxidant defense system, and metabolic pathways of carbohydrates, amino acids and carboxylic acid (Figure 6B). The 8 downregulated proteins identified by proteomics were Anxa2, Anxa1, Vcl, Cand1, Cyb5r3, Dpy30, Slc7a5, Dnajc4 that were not linked to any enriched pathway.

\section{Discussion}

Treatment with mitochondrial Kv1.3 inhibitors PAPTP and PCARBTP in an orthotopic mouse model using mouse Pan02 cells resulted in tumor growth inhibition of almost $90 \%$. When treatment was combined with cytotoxic chemotherapy Gemcitabine and Abraxane (albuminbound paclitaxel), an even greater tumor growth reduction of $95 \%$ was seen. Most importantly, this treatment strategy did not result in organ toxicity. We are not aware of a similarly successful treatment in PDAC. These studies open the possibility to identify further drug combinations that allow complete eradication of the tumor. We chose to use Gemcitabine and Abraxane as it is one of the common cytotoxic chemotherapy regimens used in the treatment of PDAC and is a 2 drug regimen in comparison to FOLFIRINOX (5-fluorouracil, oxaliplatin, irinotecan), which involves 3 drugs and associated with different toxicity profile. ${ }^{20,21}$ Although there was further reduction in tumor growth with addition of Gemcitabine/Abraxane, this study showed that there are subpopulations of tumor cells that escape cell death. Most probably, these remaining cells have an upregulated anti-oxidant defense system, as we showed at least in vitro that PCARBTP-resistant cells are characterized by this event.

The role of mitochondrial Kv1.3 in cell proliferation and apoptosis in patients with pancreas ductal adenocarcinoma is not well known. We found that Kv1.3 was highly expressed in resectable huan pancreatic cancer specimens. This is consistent with previous data from us showing by Affymetrix analysis as well as Western blots that Kv1.3 is highly expressed in 
various, largely chemoresistant human PDAC cell lines harboring p53 mutations (PANC-1, AsPC1, BxPC-3, Capan-1, Colo-357, MiaPaCa2) ${ }^{16}$. The channel is present also in the mitochondrial fraction of these cells ${ }^{22}$.

Our study demonstrates increased expression of Kv1.3 in pancreas cancer. A previous investigation by Bielanska et al. showed that Kv1.3 was under-expressed in human PDAC specimens, but these immunohistochemical studies were performed in a very limited sample $(n=2)$ of patients ${ }^{23}$. Another study examined 18 patients and found a correlation between Kv1.3 expression decrease and metastasis ${ }^{24}$. Please note that both studies were performed on tumor samples from patients with metastatic PDAC, which is biologically different than those with resectable, non-metastatic disease. Given that our study found that Kv1.3 is highly expressed in resected PDAC, it is possible that during the transition to a metastatic phenotype, pancreas cancer cells are able to down regulate Kv1.3, possibly due to methylation of the promoter region of the Kcna3 gene encoding for $\mathrm{Kv} 1.3^{24}$. The findings reported here are in agreement with previous studies showing that $\mathrm{K}^{+}$channels can promote proliferation ${ }^{25}$, while downregulation of $\mathrm{Kv} 1.3$ renders cells resistant to apoptotic stimuli ${ }^{26}$. In addition, overexpression of the channel in our patient samples suggests $\mathrm{Kv} 1.3$ is a novel therapeutic target to treat pancreas cancer. All tumor specimen showed Kv1.3 expression, at least a low general level. Even in those patient samples with low general Kv1.3 expression, there are some tumor cells that express Kv1.3 and are likely to respond to Kv1.3 inhibitors. The nature of these cells remains to be determined. It will be interesting to test whether these specific cells have some characteristics of stem cells. When examining if Kv1.3 expression holds prognostic value, we found no association with overall or recurrence free survival. It is possible that in this patient population with resectable PDAC, compared to metastatic PDAC, alterations at the DNA and/or protein level have not occurred to Kv1.3 to make it a prognostic biomarker. In this study with Western blot analysis, we did not examine mitochondrial Kv1.3 expression but rather that of Kv1.3 also found in all cellular compartments (nuclear membrane, Golgi, plasma membrane and mitochondria). What prognostic value mitochondrial Kv1.3 holds in humans remains unclear. 
We also examined if there was an association of Kv1.3 expression with presence of nodal metastases, lymphovascular invasion (LVI), and perineural invasion (PNI) and found there was none ( $p>0.05)$. This is likely due to the high rates of PNI (91\%), LVI (56\%), and positive lymph nodes $(84 \%)$ in these patients even with localized disease. The predictive value of Kv1.3 expression to PAPTP and PCARBTP treatment in mice was not tested in this study but we have previously shown a positive correlation between Kv1.3 expression and PCARBTP/PAPTPinduced death in human PDAC lines as well as between expression of the channel in mitochondria and in the plasma membrane. ${ }^{16}$

Mechanistically, the pathway of PCARBTP or PAPTP induced tumor cell death was associated with ROS production able to drive the cells over a critical point, as indicated by the finding that $\mathrm{N}$-acetyl-cysteine, a molecule able to boost the antioxidant system prevented the in vivo tumor reducing effect of both drugs ${ }^{16}$. Here we show that additional mechanisms also come into play, namely an increase in the phosphorylation of p38/MAPK and SAPK/JNK was found. When treated with the p38/SAPK inhibitor, SB203580, there was a reduction in PCARBTP-mediated in vivo effect, suggesting that mitoKv1.3 inhibition triggers, at least in part, the p38 and/or SAPKmediated death pathway. These findings are consistent with existing data that show activation of the p38/MAPK and SAPK/JNK pathways to be a favorable prognostic marker and associated with improved overall survival in patients with PDAC. ${ }^{27-29}$

Despite an unprecedented 95\% tumor growth inhibition using a combination of mitoKv1.3 inhibitors and cytotoxic chemotherapies, there were still viable tumor cells. After developing a PCARBTP treatment resistant cell line, proteomic analysis revealed there was an almost a fourfold increase in the antioxidant system (see Figure 6B). Compared to normal human cells, cancer cells have increased reactive oxygen species (ROS) generation due to reduced ability to produce scavengers. ${ }^{30,31}$ Inhibition of mitoKv1.3 can initiate a cascade of events that leads to transient hyperpolarization of the inner mitochondrial membrane, formation of ROS, stimulation of permeability transition pore, release of cytochrome c, and resulting apoptosis. $^{26,31}$. In addition, our recent findings indicate that PAP-1 derivatives bound to mitoKv1.3 are positioned in a way that their psoralenic moieties can directly accept electrons from complex I of the respiratory chain and donate these electrons to molecular oxygen, 
further boosting ROS. ${ }^{18}$ Given the importance of ROS generation to the mechanism of mitoKv1.3 inhibitor mediated cell death, it is not surprising to see that cancer cells resistant to PCARBTP upregulated the antioxidant system (GSR, GSTO1, GSTA2, for description of function see Table I). Likewise, it is not surprising that proteins linked to the cytochrome P450-mediated detoxification system are upregulated (EPHX1, GSTO1, GSTA2, ALDH3A1), as this system is mainly responsible for xenobiotic metabolism in the cells ${ }^{32}$. In addition, an upregulation of proteins linked to metabolic processes such as glycolysis, carboxylic acid biosynthesis and amino acid synthesis can be observed (see Figure 6D). This result can be interpreted as a consequence of the inhibition of the mitochondrial respiration and ATP production ${ }^{16}$. In this respect it is interesting to note that the metabolic effects of the Shk-186 toxin, that acts only on the plasma membrane located $\mathrm{Kv} 1.3^{33}$ are different from those reported here for inhibition of mitoKv1.3.

Future studies should focus on using mitoKv1.3 inhibitors to selectively kill cells with higher Kv1.3 expression, which may result in not only the direct killing of cancer cells but also enhance the activity of cytotoxic chemotherapies and potentially immunotherapies.

\section{Conclusion}

The potassium channel Kv1.3 is found to be overexpressed in pancreas adenocarcinoma and mitoKv1.3 inhibitors combined with cytotoxic chemotherapies can result in greater than 95\% tumor growth reduction without organ toxicity. In vitro western blot analysis and in vivo orthotopic model results indicated PCARBTP-mediated cell death indeed occurs through the p38 MAPK pathway. SWATH-MS and STRING and pathway enrichment analysis found the proteins linked to anti-oxidant defense system, and metabolic pathways of carbohydrates, amino acids and carboxylic acid are different in PCARBTP resistant clones. These data suggest utilizing Kv1.3 should be considered a novel therapeutic target for pancreatic cancer treatment.

\section{Materials and Methods}




\section{Orthotopic Mouse Pancreatic Tumor Injection Model}

All animal experiments were approved by University of Cincinnati Ethic Committee and Institutional Animal Care and Use Committee. Eight-week-old, wild type male, C57BL/6J mice were purchased from Jackson Labs (000664, Jackson Labs, USA). Mice were anesthetized using $120 \mathrm{mg} / \mathrm{kg}$ ketamine plus $20 \mathrm{mg} / \mathrm{kg}$ xylazine. Orthotopic injection was performed as described by Tepal et al. ${ }^{34}$ In detail, a left subcostal incision was made just below rib cage and the pancreas was identified. The tumor cell suspension was created by mixing $25 \mu \mathrm{L}$ of Matrigel with $25 \mu \mathrm{L}$ of Pan02 cells (National Cancer Institute- Frederick Cancer Research and Development Center, Frederick, MD, USA) containing $1 \times 10^{6}$ cells. Pan02 cells were cultured in DMEM $+10 \%$ FBS medium, under $37^{\circ} \mathrm{C}$ and $5 \% \mathrm{CO}_{2}$, no antibiotic added. The tumor suspension was slowly injected into the pancreas and the needle left in place for 60 seconds to allow the Matrigel to set. After ensuring hemostasis, the abdomen was closed in 2 layers using 3-0 silk suture.

\section{In Vivo Kv1.3 Inhibitor and Cytotoxic Chemotherapy Administration}

PCARBTP was employed with two kinds of application methods. For Figure $2 \mathrm{~A}, \mathrm{~B}, \mathrm{C}$ and D, PCARBTP was suspended in 50\% DMSO at concentration of $25 \mathrm{mM}$ and $50 \mathrm{mM}$ and filled in 1003D mini-pumps (MICRO-OSMOTIC PUMP MODEL 1003D, Reservoir Volume, $100 \mu \mathrm{l}, 1.0 \mu \mathrm{l}$ per hour, 3 days). The doses of PCARBTP equated to $1 \mathrm{nmol} / \mathrm{h} / \mathrm{gbw}$ and $2 \mathrm{nmol} / \mathrm{h} / \mathrm{gbw}$. The minipumps were implanted into the peritoneal cavity on day 6 after tumor injection. Pumps were replaced after drug release was completed. In the remaining experiments, PCARBTP was suspended in 50\% DMSO and injected into the peritoneal cavity at a dose of $15 \mathrm{nmol} / \mathrm{gbw}$ on day 6 after tumor injection. Similarly, PAPTP was administrated at a dose of $5 \mathrm{nmol} / \mathrm{gbw}$. Gemcitabine was dissolved in $\mathrm{dd}_{2} \mathrm{O}$ and injected into the intraperitoneal cavity at dose of 190 nmol/gbw 6 days after tumor injection. Abraxane was dissolved in DMSO and intraperitoneally injected at a dose of $23.4 \mathrm{nmol} / \mathrm{gbw}$.

\section{Orthotopic Pancreatic Tumor Transplantation Model}

Mice were injected pancreatic tumor as described as above, then treated with $15 \mathrm{nmol} / \mathrm{gbw}$ PCARBTP or $5 \mathrm{nmol} / \mathrm{gbw}$ PAPTP at day 6,8 , and 10 post tumor injection for 3 doses. Mice were kept until day 21 and the pancreatic tumors were isolated and digested with $0.25 \%$ trypsin- 
EDTA (25200056, Thermo Fisher, USA) and DNase I (DN25, Sigma, USA), single cells were collected, $1 \times 10^{6}$ primary cells in $25 \mu \mathrm{L}$ were mixed with $25 \mu \mathrm{L}$ of Matrigel and slowly injected into the pancreas of wild type C57BL/6J mice (000664, Jackson Labs, USA) as described above. The $2^{\text {nd }}$ cycle mice were treated with $15 \mathrm{nmol} / \mathrm{gbw}$ PCARBTP or $5 \mathrm{nmol} / \mathrm{gbw}$ PAPTP at day 6,8 , and 10 post tumor injection and kept until day 21 after which tumors were collected for analysis.

\section{Immunohistochemistry}

Immunohistochemistry staining was performed using a biotin-streptavidin-peroxidase (SP) kit (AB64269, Abcam, USA) and a diaminobenzidine kit (DAB) as previously described ${ }^{19}$. Institutional Review Board approval was obtained to obtain human resected pancreas ductal adenocarcinoma specimens and associated clinicopathologic data (IRB 2019-0324). Tumor specimens were obtained from the University of Cincinnati Department of Pathology. Fivemicrometer sections were deparaffinized and rehydrated in xylene and gradients of ethanol. Slides were boiled in citrate buffer $(10 \mathrm{mM}$ sodium citrate, $10 \mathrm{mM}$ citric acid, $\mathrm{pH} 6.0)$ at $92-98^{\circ} \mathrm{C}$ for 10 min to retrieve the antigen. The sections were then incubated with $3 \% \mathrm{H}_{2} \mathrm{O}_{2}$ in methanol for $10 \mathrm{~min}$ to quench endogenous peroxidase and blocked with normal goat serum for $20 \mathrm{~min}$. Sections were incubated with specific primary antibodies against Kv1.3 (1 : 200; P4497, Sigma , USA) at $4^{\circ} \mathrm{C}$ overnight. The sections were then incubated with biotinylated goat-anti-rabbit IgG secondary antibody and sections stained with DAB working reagent (per manufacturer's instructions) for 30-60 seconds. They were then counterstained with hematoxylin. Finally, sections were mounted with Permount (SP15-500, Fisher, USA) onto slides. Negative control was performed using unconjugated rabbit IgG (011-000-003, Jackson ImmunoResearch Laboratories, Inc., West Grove, PA, USA). Images were acquired with a ZEISS AXIO microscope (Carl ZEISS, Germany). Slides were scored by a gastrointestinal pathologist who specializes in evaluating pancreas cancer. He was blinded to all clinicopathologic data and determined percent and intensity of staining. A final score of high versus low expression were obtained based on these data.

\section{Western Blot}


Cells were lysed in whole-cell lysis buffer (50 mM HEPES, 150 mM NaCl, 1 mM EGTA, 10 mM sodium pyrophosphatate, $1.5 \mathrm{mM} \mathrm{MgCl}_{2}, 100 \mathrm{mM} \mathrm{NaF}, 10 \%$ glycerol and 1\% Triton X-100, pH 7.2) containing an inhibitor cocktail ( $1 \mathrm{mM}$ phenylmethylsulfonyl fluoride, $10 \mathrm{mg} / \mathrm{ml}$ aprotinin and $1 \mathrm{mM}$ sodium orthovanadate) to extract total protein. Protein concentrations were determined using a standard bicinchoninic acid (BCA) assay (23225, Thermo Fisher Scientific Inc., Waltham, MA, USA), and $50 \mu \mathrm{g}$ of total protein was subjected to $10 \%$ SDS-PAGE followed by electrotransfer onto nitrocellulose membranes. The membranes were blocked in $5 \%$ skim milk, and then incubated overnight at $4{ }^{\circ} \mathrm{C}$ with primary antibodies against human Kv1.3 (1 : 1000, P4497, Sigma, USA), P-p38 MAPK (1 : 1000; Cell signaling), P-SAPK/JNK (1 : 1000; Cell signaling), $\beta$-actin (1: 2 000; Abcam). Membranes were then washed with TBST for $3 \times 10$ min. This was followed by incubation with horseradish peroxidase-conjugated secondary antibodies for $1 \mathrm{~h}$ at room temperature in 5\% skim milk and washed with TBST for $3 \times 10 \mathrm{~min}$. Immunoreactive signals were detected using enhanced chemiluminescence (Pierce, Rockford, IL, USA). Three independent experiments were performed.

\section{H\&E Staining}

Slides containing paraffin sections were passed using the following steps: 3 x $5 \mathrm{~min}$ in Xylene (blot excess xylene before going into ethanol), 2 x 5min in 100\% ethanol, 1 x 5min in 95\% ethanol, $1 \times 5 \mathrm{~min}$ in $70 \%$ ethanol, $1 \times 5 \mathrm{~min}$ deionized $\mathrm{H}_{2} \mathrm{O}$, and $1 \times 3$ min Hematoxylin. They were then rinsed with deionized water $1 \times 5 \mathrm{~min}$, tap water and ethanol to destain. After subsequent rinse, they were treated $1 \times 30 \mathrm{sec}$ Eosin, $3 \times 5 \mathrm{~min} 95 \%$ ethanol, $3 \times 5 \mathrm{~min} 100 \%$ ethanol, 3 x 5min Xylene, and coverslip was placed using Permount mounting medium (SP15500, Fisher, USA).

\section{PCARBTP Resistant Clones}

Pan02 cells were detached from tissue culture flasks before reaching confluence by removing the culture medium, adding trypsin-EDTA and incubating for $3 \mathrm{~min}$ at $37^{\circ} \mathrm{C}$ and $5 \% \mathrm{CO}_{2}$. After this incubation period, fresh medium was added and cells were spun at $800 \mathrm{rpm}$ for $5 \mathrm{~min}$. Supernatant was removed and fresh medium added. Cell count was carried out with the standard trypan blue exclusion method. Pan02 cells were seeded at 1 cell per well in $400 \mu \mathrm{L}$ of 
fresh medium in 96 flat-bottomed well plates. Clones were inspected regularly so those wells with more than 1 clone could be discarded. The addition of PCARBTP was carried out by replacing medium containing PCARBTP at the different doses. If the cells survived at that dose for more than 3 days, we increased the dose, and obtained the 4 clones of Pan02 cells that survived under $10 \mu \mathrm{M}$ PCARBTP medium. Resistant cells were amplified under $10 \mu \mathrm{M}$ PCARBTP then proteins were collected for proteomic analysis.

Proteomic Analysis of Resistant Clones The Pierce 660nm Protein assay was performed on a 1:10 dilution of the samples to determine the protein concentration using BSA as a standard. Sufficient protein was present such that $50 \mu \mathrm{g}$ was taken out to run on a short 1D gel for digestion. $50 \mu \mathrm{g}$ of each sample (non-resistant and resistant clones) in $40 \mu \mathrm{L}$ of Laemmli buffer were run $1.5 \mathrm{~cm}$ into a 1D, $1.5 \mathrm{~mm} \mathrm{4-12 \%} \mathrm{BT} \mathrm{gel} \mathrm{using} \mathrm{MOPS} \mathrm{running} \mathrm{buffer.} \mathrm{Pre-stained}$ protein markers were used in surrounding lanes. The regions between the markers and the dye front were excised for trypsin digestion following the standard in gel protocol. The resulting peptides were extracted, dried, and prepared for mass spectrometry. $2.5 \mu \mathrm{g}$ of each sample was run on the nanoLC-MS/MS in DDA mode and the combined DDA runs were searched using Protein Pilot (SCIEX, AB Sciex Pte. Ltd, USA) to create the protein spectral library. 720 proteins were identified with $99 \%$ confidence with an FDR of less than $1 \%$ at peptide and protein level. A matched SWATH-MS method in DIA mode of the samples was used to collect quantitative data for each of the samples for the comparative profiling. Three clones of PCARBTP resistant cells and three replicates of normal Pan02 were processed. SWATH-D data analysis workflow was used to validate the data set and detected significant quantitative changes.

\section{Statistical Analysis}

Clinicopathologic data were obtained from the electronic medical records. Variables include patient age, gender, histologic grade, lymphovascular invasion, perineural invasion, stage, lymph node status, and oncologic status. Statistical analyses were performed using SPSS 26 (IBM Inc., Armonk, NY). Descriptive statistics are reported as the median and interquartile 
range (IQR). Chi-squared tests or Fisher's exact test were used for comparing categorical variables. Median values or continuous variables were examined using Student's t-test or Mann-Whitney. Survival analyses were performed using Kaplan-Meier methodology. P-values $<0.05$ were considered statistically significant. The tumor volume and mass and mouse body weight were analysed by t-test and one-way ANOVA using GraphPad Prism 9.0, each experiment has more than 6 mice in one group indicated in Results and Figure legends. $\mathrm{P}<0.05$ was considered as statistically significant. ${ }^{*} p<0.05 ;{ }^{*} p<0.01, * * * p<0.0005, * * * * p<0.0001$.

\section{Acknowledgments}

The authors would like to thank the Boyce Family Foundation for their support in this research endeavor. This study was funded, in part, by the Central Surgical Association Turcotte Award, 2019 and in part by the Italian Association for Cancer Research (IG 20286). The authors thank Dr.s Lucia Biasutto, Sofia Parrasia and Andrea Rossa for help with chemical analysis of the $\mathrm{K}^{+}$ channel inhibitors used in this study and Dr. Mario Zoratti for useful discussion and critical reading of the manuscript.

\section{Authors' Contributions}

Conception and design: $W L, M B, M J E, I S, C P, A M, E G, S A A$, and SHP

Development of methodology: WL, MB, MJE, GCW, CP, AM, JW, IS, EG, SAA, and SHP

Acquisition of data (provided animals, acquired and managed patients, provided facilities, etc.): WL, MB, GCW, CP, AM, JW, EG, SAA, and SHP

Analysis and interpretation of data (e.g., statistical analysis, biostatistics, computational analysis): WL, MB, MJE, GCW, JW, CP, AM, IS, EG, SAA, and SHP

Writing, review, and/or revision of the manuscript: $W L, M B, M J E, G C W, C P, A M, J W, I S, E G$, SAA, and SHP

Administrative, technical, or material support (i.e., reporting or organizing data, constructing databases): WL, EG, and SHP

Study supervision: WL, MB, MJE, GCW, EG, SAA, and SHP 
Other (provided intellectual input and edited the article): WL, MB, MJE, GCW, CP, AM, JW, IS, EG, SAA, and SHP

\section{Conflict of Interest}

The authors have no conflicts of interest to disclose

\section{The Paper Explained}

Problem Treatment of pancreas ductal adenocarcinoma (PDAC) remains challenging due to the late stage of presentation, limited efficacy of cytotoxic chemotherapies, and aggressive tumor biology. Novel therapeutic targets are desperately needed. The voltage-gated potassium channel, Kv1.3, is one such unique target. It has been extensively studied in many cancers but less is known in pancreas cancer. In this study we evaluated tissue expression of Kv1.3 in resected PDAC and tumor inhibition using novel Kv1.3 inhibitors developed by our group (PCARBTP and PAPTP).

Results We found that in the largest cohort of surgically resected PDAC specimens, Kv1.3 exhibits high tissue expression in over half of tumors. Utilizing orthotopic pancreas tumor mouse models, treatment with mitochondrial Kv1.3 inhibitors, PCARBTP and PAPTP resulted in tumor reductions of $87 \%$ and $70 \%$, respectively. When these inhibitors were combined with cytotoxic chemotherapies (gemcitabine/abraxane), this resulted in a $95 \%$ tumor growth inhibition without organ toxicity. Evaluation of the mechanism of cell death using in vivo models showed an integral role of the p38-MAPK pathway. Finally, resistant clones to 
mitochondrial Kv1.3 inhibitors escaped cell death through upregulation of the anti-oxidant system.

Impact The findings from this study indicate that Kv1.3 is expressed in early stage, nonmetastatic, resectable pancreas cancer specimens. Treatment with novel mitochondrial Kv1.3 inhibitors resulted in $95 \%$ reduced tumor growth when combined with cytotoxic chemotherapies. This near complete eradication of tumors using this treatment strategy shows that Kv1.3 represents an innovative therapeutic target for pancreas cancer therapy.

\section{References}

1. Ito $\mathrm{H}$, Ito $\mathrm{K}$, D'Angelica $\mathrm{M}$, et al. Accurate staging for gallbladder cancer: implications for surgical therapy and pathological assessment. Annals of surgery. 2011;254(2):320-325.

2. Siegel RL, Miller KD, Jemal A. Cancer statistics, 2020. CA: a cancer journal for clinicians. 2020;70(1):7-30.

3. Tan E, El-Rayes B. Pancreatic Cancer and Immunotherapy: Resistance Mechanisms and Proposed Solutions. Journal of gastrointestinal cancer. 2019;50(1):1-8.

4. Amedei A, Niccolai E, Prisco D. Pancreatic cancer: role of the immune system in cancer progression and vaccine-based immunotherapy. Human vaccines \& immunotherapeutics. 2014;10(11):3354-3368.

5. Comes N, Bielanska J, Vallejo-Gracia A, et al. The voltage-dependent $\mathrm{K}(+)$ channels Kv1.3 and Kv1.5 in human cancer. Frontiers in physiology. 2013;4:283.

6. Szabo I, Zoratti M, Gulbins E. Contribution of voltage-gated potassium channels to the regulation of apoptosis. FEBS letters. 2010;584(10):2049-2056.

7. Cahalan MD, Chandy KG, DeCoursey TE, Gupta S. A voltage-gated potassium channel in human T lymphocytes. The Journal of physiology. 1985;358:197-237.

8. Matteson DR, Deutsch C. K channels in T lymphocytes: a patch clamp study using monoclonal antibody adhesion. Nature. 1984;307(5950):468-471.

9. Wulff $\mathrm{H}$, Castle NA, Pardo LA. Voltage-gated potassium channels as therapeutic targets. Nature reviews Drug discovery. 2009;8(12):982-1001.

10. Panyi G, Possani LD, Rodriguez de la Vega RC, Gaspar R, Varga Z. K+ channel blockers: novel tools to inhibit $\mathrm{T}$ cell activation leading to specific immunosuppression. Current pharmaceutical design. 2006;12(18):2199-2220.

11. Cahalan MD, Chandy KG. The functional network of ion channels in T lymphocytes. Immunol Rev. 2009;231(1):59-87.

12. Feske $\mathrm{S}$, Wulff $\mathrm{H}$, Skolnik EY. Ion channels in innate and adaptive immunity. Annual review of immunology. 2015;33:291-353.

13. Perez-Verdaguer M, Capera J, Serrano-Novillo C, Estadella I, Sastre D, Felipe A. The voltagegated potassium channel Kv1.3 is a promising multitherapeutic target against human pathologies. Expert opinion on therapeutic targets. 2016;20(5):577-591. 
14. Chandy KG, Norton RS. Peptide blockers of Kv1.3 channels in T cells as therapeutics for autoimmune disease. Current opinion in chemical biology. 2017;38:97-107.

15. Serrano-Albarras A, Estadella I, Cirera-Rocosa S, Navarro-Perez M, Felipe A. Kv1.3: a multifunctional channel with many pathological implications. Expert opinion on therapeutic targets. 2018;22(2):101-105.

16. Leanza L, Romio M, Becker KA, et al. Direct Pharmacological Targeting of a Mitochondrial Ion Channel Selectively Kills Tumor Cells In Vivo. Cancer cell. 2017;31(4):516-531.e510.

17. Leanza L, Henry B, Sassi N, et al. Inhibitors of mitochondrial Kv1.3 channels induce Bax/Bakindependent death of cancer cells. EMBO molecular medicine. 2012;4(7):577-593.

18. Peruzzo $R$, Mattarei $A$, Azzolini $M$, et al. Insight into the mechanism of cytotoxicity of membrane-permeant psoralenic Kv1.3 channel inhibitors by chemical dissection of a novel member of the family. Redox biology. 2020;37:101705.

19. Bergermann T, Born L, Ferguson F, et al. Inhibition of PI-3-K and AKT Amplifies Kv1.3 InhibitorInduced Death of Human T Leukemia Cells. Cellular physiology and biochemistry : international journal of experimental cellular physiology, biochemistry, and pharmacology. 2019;53(S1):1-10.

20. Von Hoff DD, Ervin T, Arena FP, et al. Increased survival in pancreatic cancer with nab-paclitaxel plus gemcitabine. The New England journal of medicine. 2013;369(18):1691-1703.

21. Sohal D, McDonough S, Ahmad SA, et al. SWOG S1505: Initial findings on eligibility and neoadjuvant chemotherapy experience with mfolfirinox versus gemcitabine/nab-paclitaxel for resectable pancreatic adenocarcinoma. Journal of Clinical Oncology. 2019;37(4_suppl):414-414.

22. Zaccagnino A, Manago A, Leanza L, et al. Tumor-reducing effect of the clinically used drug clofazimine in a SCID mouse model of pancreatic ductal adenocarcinoma. Oncotarget. 2016.

23. Bielanska J, Hernandez-Losa J, Perez-Verdaguer $\mathrm{M}$, et al. Voltage-dependent potassium channels Kv1.3 and Kv1.5 in human cancer. Current cancer drug targets. 2009;9(8):904-914.

24. Brevet $M$, Fucks $D$, Chatelain $D$, et al. Deregulation of 2 potassium channels in pancreas adenocarcinomas: implication of KV1.3 gene promoter methylation. Pancreas. 2009;38(6):649654.

25. Pardo LA, Stuhmer W. The roles of $\mathrm{K}(+)$ channels in cancer. Nature reviews Cancer. 2014;14(1):39-48.

26. Szabo I, Bock J, Grassme H, et al. Mitochondrial potassium channel Kv1.3 mediates Bax-induced apoptosis in lymphocytes. Proceedings of the National Academy of Sciences of the United States of America. 2008;105(39):14861-14866.

27. Zhong Y, Naito Y, Cope L, et al. Functional p38 MAPK identified by biomarker profiling of pancreatic cancer restrains growth through JNK inhibition and correlates with improved survival. Clinical cancer research : an official journal of the American Association for Cancer Research. 2014;20(23):6200-6211.

28. Zhang L, Fang Y, Xu XF, Jin DY. Moscatilin induces apoptosis of pancreatic cancer cells via reactive oxygen species and the JNK/SAPK pathway. Molecular medicine reports. 2017;15(3):1195-1203.

29. Qian CJ, Qi YX, Zhong S, Zeng JP, Chen XY, Yao J. Mitogen-activated protein kinase inhibition enhances the antitumor effects of sporamin in human pancreatic cancer cells. Oncology letters. 2018;16(1):1237-1242.

30. Gorrini C, Harris IS, Mak TW. Modulation of oxidative stress as an anticancer strategy. Nature reviews Drug discovery. 2013;12(12):931-947.

31. Checchetto V, Prosdocimi E, Leanza L. Mitochondrial Kv1.3: a New Target in Cancer Biology? Cellular physiology and biochemistry : international journal of experimental cellular physiology, biochemistry, and pharmacology. 2019;53(S1):52-62. 
32. Waring RH. Cytochrome P450: genotype to phenotype. Xenobiotica; the fate of foreign compounds in biological systems. 2020;50(1):9-18.

33. Upadhyay SK, Eckel-Mahan KL, Mirbolooki MR, et al. Selective Kv1.3 channel blocker as therapeutic for obesity and insulin resistance. Proceedings of the National Academy of Sciences of the United States of America. 2013;110(24):E2239-2248.

34. Tepel J, Dagvadorj O, Kapischke M, et al. Significant growth inhibition of orthotopic pancreatic ductal adenocarcinoma by $\mathrm{CpG}$ oligonucleotides in immunodeficient mice. Int J Colorectal Dis. 2006;21(4):365-372.

\section{Figure Legends:}

Figure 1. Expression of Kv1.3 in human pancreatic cancer tissue and pancreatic cell lines.

A. Expression of Kv1.3 in human pancreatic tissue. Panel a, high expression level of Kv1.3 in human pancreatic cancer tissue. ( $n=33$, scale bar=50 $\mu \mathrm{m})$. Panel $b$, magnification of panel $a$, scale bar $=20 \mu \mathrm{m}$. Panel c, low expression level of Kv1.3 in human pancreatic cancer tissue. $(n=22$, scale bar=50 $\mu \mathrm{m})$. Panel d, magnification of panel c, scale bar=20 $\mu \mathrm{m}$. Panel e, IgG control with magnification (Panel f). Panel $g$ and $h$ show high expression of Kv1.3 and an example of a normal pancreas duct (black arrow).

B, Recurrence free survival and C, overall survival based on Kv1.3 expression in resected human pancreatic cancer tissues.

Figure 2. PCARBTP reduced pancreatic tumor size in mouse models through mini-pump administration.

A. PCARBTP reduced mouse pancreatic tumor volume orthotopically significantly by mini-pump application method, dose of PCARBTP was $1 \mathrm{nmol} / \mathrm{h} / \mathrm{gbw}$ and $2 \mathrm{nmol} / \mathrm{h} / \mathrm{gbw}$. PCARBTP reduced 
mouse pancreatic tumor weight orthotopically significantly by mini-pump application method, $\mathrm{n}=7$. B. Bodyweights change of the mice during PCARBTP treatment were no difference $(\mathrm{p}=0.84$ for $1 \mathrm{nmol} / \mathrm{h} / \mathrm{gbw}$ and $\mathrm{p}=0.22$ for $2 \mathrm{nmol} / \mathrm{h} / \mathrm{gbw})$. C. Representative pictures of the mouse orthotopically injected tumors, $n=7$.

Table 1. Treatment Regimen of Figure $2 \mathrm{~A}-\mathrm{C}$.

Figure 3. PCARBTP reduced pancreatic tumor size in mouse models through intraperitoneal administration.

A. PCARBTP reduced mouse pancreatic tumor volume orthotopically significantly by intraperitoneal administration, $\mathrm{n}=8$. PCARBTP reduced mouse pancreatic tumor weight orthotopically significantly by intraperitoneal administration. B. Bodyweights change of the mice during PCARBTP treatment were less than $20 \%(p<0.05)$. C. Representative pictures of the mouse orthotopically injected tumors.

Table 2. Treatment Regimen of Figure 3 A-C.

Figure 4. PAPTP reduced pancreatic tumor size in mouse models through intraperitoneal administration.

A. PAPTP reduced mouse pancreatic tumor volume orthotopically significantly by intraperitoneal administration, $n=8$. PAPTP reduced mouse pancreatic tumor weight orthotopically significantly by intraperitoneal administration, $n=8$. B. Bodyweights change of the mice during PAPTP treatment were no difference $(p=0.17)$. C. Representative pictures of the mouse orthotopically injected tumors.

Table 3. Treatment Regimen of Figure $4 \mathrm{~A}-\mathrm{C}$. 
Figure 5. PCARBTP or PAPTP combined with Gemcitabine and Abraxane reduced pancreatic tumor size and weight in mouse model.

A. PCARBTP worked better than PAPTP, and PCARBTP combined with Gemcitabine and Abraxane reduced $95 \%$ the pancreas tumor volume. ${ }^{*} p<0.0001$, compared to the control group. $n=8$.

B. Weight of the tumors same with panel A. ${ }^{*} p<0.0001$, compared to the control group. $n=8$.

Table 4. Treatment Regimen of Figure 3.

C. Representative pictures of the mouse orthotopically injected tumors.

Table 4. Treatment Regimen of Figure 3.

Figure 6. PCARBTP/PAPTP toxic on other organs in mouse model. H\&E staining showed either PCARBTP or PAPTP intraperitoneal administration only or combined with Gemcitabine and Abraxane had no toxic effective on heart, lung, kidney and liver. Scale bar $=20 \mu \mathrm{m}$.

Figure 7. PCARBTP mediated the cell apoptosis through the p38 MAPK pathway.

A. Phosphorylation levels of p38 MAPK (Thr180/Tyr182), P-SAPK/JNK (Thr183/Tyr185) were increased during the PCARBTP treatment for $30 \mathrm{~min}$ and by dose-dependent way, $\mathrm{n}=3$. B. p38 MAPK inhibitor (SB203580) attenuated the function of PCARBTP and PCARBTP combined with Gemcitabine and Abraxane, $\mathrm{n}=6 .{ }^{*} \mathrm{p}<0.05 ; *{ }^{*} \mathrm{p}<0.01, * * * \mathrm{p}<0.0005, * * * * \mathrm{p}<0.0001$.

Table 5. Treatment Regimen of Figure 5B.

Figure 8. Drug resistance to PCARBTP developed by pancreatic tumors

A. Proteomic analysis of protein changes in PCARBTP resistant clones. Heatmap of unbiased clustering of only the significant proteins, 135 proteins with ANOVA $p<0.05$ (color in Log2- 
scale), n=3. B. STRING analysis showing the following enriched KEGG pathway-linked proteins. Number of nodes: 45; number of edges: 89; average node degree: 3.96; avg. local clustering coefficient: 0.521; expected number of edges: 23; PPI enrichment p-value:< 1.0e-16. KEGG Pathways: Light green: Glycolysis / Gluconeogenesis (ㄴa00010) (p value 3.45e-08); Yellow: Metabolic pathways (ha01100) (p value 4.26e-07); Light blue: Carbon metabolism (hsa01200) ( $p$ value 1.08e-05); Dark green Biosynthesis of amino acids (hsa01230) (p value 2.10e-05); Magenta: HIF-1 signaling pathway (hsa04066) (p value 7.12e-05); Red: Metabolism of xenobiotics by cytochrome P450 (hsa00980) ( $p$ value 0.00039); Dark blue: Glutathione metabolism ( $\underline{\text { hsa00480) }}$ ( $p$ value 0.0029).

Table I. SWATH nanoLC-MS/MS analysis of protein expression in PCARBTP-resistant Pan02 clones compared to normal Pan02 cells.

TABLE I. List of upregulated proteins and their function. Source: STRING analysis (https://stringdb.org/cgi/input.pl?taskld= notask\&sessionld=SF25ZH1QUDpd)

ARF5

ADP-ribosylation factor 5; GTP-binding protein that functions as an allosteric activator of the cholera toxin catalytic subunit, an ADP- ribosyltransferase. Involved in protein trafficking; may modulate vesicle budding and uncoating within the Golgi apparatus; ARF GTPase family (180 aa)

Phosphatidylinositol $\mathrm{N}$-acetylglucosaminyltransferase subunit $\mathrm{Q}$; Part of the PIGQ complex catalyzing the transfer of $\mathrm{N}$ - acetylglucosamine from UDP-Nacetylglucosamine to phosphatidylinositol, the first step of GPI biosynthesis; Belongs to the PIGQ family (760 aa)

MIF Macrophage migration inhibitory factor; Pro-inflammatory cytokine. Involved in the 
innate immune response to bacterial pathogens. The expression of MIF at sites of inflammation suggests a role as mediator in regulating the function of macrophages in host defense. Counteracts the anti- inflammatory activity of glucocorticoids. Has phenylpyruvate tautomerase and dopachrome tautomerase activity (in vitro), but the physiological substrate is not known. It is not clear whether the tautomerase activity has any physiological relevance, and whether it is important for cytokine activity (115 aa)

Eukaryotic translation initiation factor 5; Catalyzes the hydrolysis of GTP bound to the $40 \mathrm{~S}$ ribosomal initiation complex (40S.mRNA.Met-tRNA[F].elF-2.GTP) with the subsequent joining of a $60 \mathrm{~S}$ ribosomal subunit resulting in the release of elF-2 and the guanine nucleotide. The subsequent joining of a 605 ribosomal subunit results in the formation of a functional $80 \mathrm{~S}$ initiation complex (80S.mRNA.Met-tRNA[F]) (431 aa)

Glycogen phosphorylase, brain form; Glycogen phosphorylase that regulates glycogen mobilization. Phosphorylase is an important allosteric enzyme in

PYGB carbohydrate metabolism. Enzymes from different sources differ in their regulatory mechanisms and in their natural substrates. However, all known phosphorylases share catalytic and structural properties (843 aa)

GSR Glutathione reductase, mitochondrial; Maintains high levels of reduced glutathione in the cytosol (522 aa)

PLIN3 Perilipin-3; Required for the transport of mannose 6-phosphate receptors (MPR) from endosomes to the trans-Golgi network; Perilipins (434 aa)

TPI1 Triosephosphate isomerase 1; Belongs to the triosephosphate isomerase family (286 aа)

ATP-dependent RNA helicase DDX39A; Isoform 1- Involved in pre-mRNA splicing.

DDX39A Required for the export of mRNA out of the nucleus; Belongs to the DEAD box helicase family. DECD subfamily (427 aa)

ATP-citrate synthase; ATP-citrate synthase is the primary enzyme responsible for the synthesis of cytosolic acetyl-CoA in many tissues. Has a central role in de novo ACLY lipid synthesis. In nervous tissue it may be involved in the biosynthesis of acetylcholine; In the $\mathrm{N}$-terminal section; belongs to the succinate/malate CoA ligase beta subunit family (1101 aa)

$\mathrm{ALDH} 2$

Aldehyde dehydrogenase, mitochondrial; Aldehyde dehydrogenase 2 family member; Belongs to the aldehyde dehydrogenase family (517 aa) 
ANXA3 properties. Also cleaves the cyclic bond of inositol 1,2-cyclic phosphate to form inositol 1-phosphate; Annexins (323 aa)

Fatty acid synthase; Fatty acid synthetase catalyzes the formation of long- chain FASN fatty acids from acetyl-CoA, malonyl-CoA and NADPH. This multifunctional protein has 7 catalytic activities and an acyl carrier protein; Seven-beta-strand methyltransferase motif containing (2511 aa)

14-3-3 protein gamma; Adapter protein implicated in the regulation of a large spectrum of both general and specialized signaling pathways. Binds to a large YWHAG number of partners, usually by recognition of a phosphoserine or phosphothreonine motif. Binding generally results in the modulation of the activity of the binding partner; Belongs to the 14-3-3 family (247 aa)

265 proteasome non-ATPase regulatory subunit 2; Component of the 265 proteasome, a multiprotein complex involved in the ATP-dependent degradation of ubiquitinated proteins. This complex plays a key role in the maintenance of protein

PSMD2 homeostasis by removing misfolded or damaged proteins, which could impair cellular functions, and by removing proteins whose functions are no longer required. Therefore, the proteasome participates in numerous cellular processes, including cell cycle progression, apoptosis, or DNA damage repair; Belongs to the proteasome subunit S2 family (908 aa)

Heat shock protein $105 \mathrm{kDa}$; Acts as a nucleotide-exchange factor (NEF) for chaperone proteins HSPA1A and HSPA1B, promoting the release of ADP from HSPA1A/B thereby triggering client/substrate protein release. Prevents the HSPH1 aggregation of denatured proteins in cells under severe stress, on which the ATP levels decrease markedly. Inhibits HSPA8/HSC70 ATPase and chaperone activities (By similarity); Heat shock 70kDa proteins (858 aa)

UGDH UDP-glucose 6-dehydrogenase; Involved in the biosynthesis of glycosaminoglycans; hyaluronan, chondroitin sulfate, and heparan sulfate (494 aa)

Acetoacetyl-CoA synthetase; Activates acetoacetate to acetoacetyl-CoA. May be involved in utilizing ketone body for the fatty acid-synthesis during adipose tissue development (By similarity); Belongs to the ATP-dependent AMP-binding enzyme family (672 aa)

Keratin, type II cytoskeletal 7; Blocks interferon-dependent interphase and KRT7 stimulates DNA synthesis in cells. Involved in the translational regulation of the human papillomavirus type 16 E7 mRNA (HPV16 E7); Keratins, type II (469 aa) 
RANBP3

Ran-binding protein 3; Acts as a cofactor for XPO1/CRM1-mediated nuclear export, perhaps as export complex scaffolding protein. Bound to XPO1/CRM1, stabilizes the XPO1/CRM1-cargo interaction. In the absence of Ran-bound GTP prevents binding of XPO1/CRM1 to the nuclear pore complex. Binds to CHC1/RCC1 and increases the guanine nucleotide exchange activity of CHC1/RCC1. Recruits XPO1/CRM1 to CHC1/RCC1 in a Ran-dependent manner. Negative regulator of TGF- beta signaling through interaction with the R-SMAD proteins, SMAD2 and SMAD3, and mediating their nuclear export (567 aa)

Heterogeneous nuclear ribonucleoprotein A1; Involved in the packaging of preHNRNPA1 mRNA into hnRNP particles, transport of poly(A) mRNA from the nucleus to the cytoplasm and may modulate splice site selection. May bind to specific miRNA hairpins; RNA binding motif containing (372 aa)

Peroxiredoxin-6; Thiol-specific peroxidase that catalyzes the reduction of hydrogen peroxide and organic hydroperoxides to water and alcohols, respectively. Can reduce $\mathrm{H}(2) \mathrm{O}(2)$ and short chain organic, fatty acid, and phospholipid

PRDX6 hydroperoxides. Also has phospholipase activity, and can therefore either reduce the oxidized sn-2 fatty acyl grup of phospholipids (peroxidase activity) or hydrolyze the sn-2 ester bond of phospholipids (phospholipase activity). These activities are dependent on binding to phospholipids at acidic $\mathrm{pH}$ and to oxidized phospholipds at cytosolic $\mathrm{pH}$. Plays a role [...] (224 aa)

Synaptic vesicle membrane protein VAT-1 homolog; Possesses ATPase activity (By

VAT1 similarity). Plays a part in calcium-regulated keratinocyte activation in epidermal repair mechanisms. Has no effect on cell proliferation. Negatively regulates mitochondrial fusion in cooperation with mitofusin proteins (MFN1-2) (393 aa)

Transferrin receptor protein 1; Cellular uptake of iron occurs via receptor-mediated endocytosis of ligand-occupied transferrin receptor into specialized endosomes. Endosomal acidification leads to iron release. The apotransferrin-receptor complex

TFRC is then recycled to the cell surface with a return to neutral $\mathrm{pH}$ and the concomitant loss of affinity of apotransferrin for its receptor. Transferrin receptor is necessary for development of erythrocytes and the nervous system (By similarity). A second ligand, the heditary hemochromatosis protein HFE, competes for binding with transferrin for [...] (760 aa)

Keratin, type I cytoskeletal 19; Involved in the organization of myofibers. Together KRT19 with KRT8, helps to link the contractile apparatus to dystrophin at the costameres of striated muscle; Keratins, type I (400 aa)

EZR Ezrin; Probably involved in connections of major cytoskeletal structures to the 
plasma membrane. In epithelial cells, required for the formation of microvilli and membrane ruffles on the apical pole. Along with PLEKHG6, required for normal macropinocytosis; A-kinase anchoring proteins (586 aa)

D-3-phosphoglycerate dehydrogenase; Catalyzes the reversible oxidation of 3phospho-D- glycerate to 3-phosphonooxypyruvate, the first step of the PHGDH phosphorylated L-serine biosynthesis pathway. Also catalyzes the reversible oxidation of 2-hydroxyglutarate to 2-oxoglutarate and the reversible oxidation of (S)-malate to oxaloacetate; Belongs to the D-isomer specific 2-hydroxyacid dehydrogenase family (533 aa)

Glutathione S-transferase omega-1; Exhibits glutathione-dependent thiol transferase and dehydroascorbate reductase activities. Has S-(phenacyl)glutathione

GSTO1 reductase activity. Has also glutathione S-transferase activity. Participates in the biotransformation of inorganic arsenic and reduces monomethylarsonic acid (MMA) and dimethylarsonic acid; Soluble glutathione S-transferases (241 aa)

PGM1 Phosphoglucomutase-1; This enzyme participates in both the breakdown and synthesis of glucose; Belongs to the phosphohexose mutase family (580 aa)

Phosphoglycerate kinase $1 ;$ In addition to its role as a glycolytic enzyme, it seems PGK1 that PGK-1 acts as a polymerase alpha cofactor protein (primer recognition protein). May play a role in sperm motility (417 aa)

ESD

S-formylglutathione hydrolase; Serine hydrolase involved in the detoxification of formaldehyde (282 aa)

Xanthine dehydrogenase/oxidase; Key enzyme in purine degradation. Catalyzes the $\mathrm{XDH}$ oxidation of hypoxanthine to xanthine. Catalyzes the oxidation of xanthine to uric acid. Contributes to the generation of reactive oxygen species. Has also low oxidase activity towards aldehydes (in vitro) (1333 aa)

Guanine nucleotide-binding protein $G(I) / G(S) / G(O)$ subunit gamma-7; Guanine nucleotide-binding proteins ( $G$ proteins) are involved as a modulator or transducer in various transmembrane signaling systems. The beta and gamma chains are GNG7 required for the GTPase activity, for replacement of GDP by GTP, and for G proteineffector interaction. Plays a role in the regulation of adenylyl cyclase signaling in certain regions of the brain. Plays a role in the formation or stabilzation of a $G$ protein heterotrimer (G(olf) subunit alpha-beta-gamma-7) that is required for adenylyl cyclase activity in [...] (68 aa)

ALDOA Fructose-bisphosphate aldolase $A$; Plays a key role in glycolysis and gluconeogenesis. In addition, may also function as scaffolding protein (By 
similarity); Belongs to the class I fructose-bisphosphate aldolase family (418 aa)

Phosphoserine phosphatase; Catalyzes the last step in the biosynthesis of serine PSPH from carbohydrates. The reaction mechanism proceeds via the formation of a phosphoryl-enzyme intermediates; HAD Asp-based non-protein phosphatases (225 aa)

$\mathrm{HIC2}$

Hypermethylated in cancer 2 protein; Transcriptional repressor; BTB domain containing (615 aa)

Aldehyde dehydrogenase, dimeric NADP-preferring; ALDHs play a major role in the detoxification of alcohol-derived acetaldehyde (Probable). They are involved in the metabolism of corticosteroids, biogenic amines, neurotransmitters, and lipid

ALDH3A1 peroxidation (Probable). Oxidizes medium and long chain aldehydes into non-toxic fatty acids. Preferentially oxidizes aromatic aldehyde substrates. Comprises about 50 percent of corneal epithelial soluble proteins (By similarity). May play a role in preventing corneal damage caused by ultraviolet light (By similarity) (453 aa)

Solute carrier family 2, facilitated glucose transporter member 1; Facilitative glucose transporter. This isoform may be responsible for constitutive or basal

SLC2A1 glucose uptake. Has a very broad substrate specificity; can transport a wide range of aldoses including both pentoses and hexoses; Belongs to the major facilitator superfamily. Sugar transporter (TC 2.A.1.1) family. Glucose transporter subfamily (492 аa)

NADPH--cytochrome P450 reductase; This enzyme is required for electron transfer POR from NADP to cytochrome P450 in microsomes. It can also provide electron transfer to heme oxygenase and cytochrome B5; Belongs to the NADPH--cytochrome P450 reductase family (680 aa)

Glutathione S-transferase A2; Conjugation of reduced glutathione to a wide number GSTA2 of exogenous and endogenous hydrophobic electrophiles; Belongs to the GST superfamily. Alpha family (222 aa)

HARS

Histidine--tRNA ligase, cytoplasmic; Cytoplasmic histidine--tRNA ligase (Probable). Plays a role in axon guidance; Aminoacyl tRNA synthetases, Class II (509 aa)

General vesicular transport factor p115; General vesicular transport factor required for intercisternal transport in the Golgi stack; it is required for transcytotic fusion USO1 and/or subsequent binding of the vesicles to the target membrane. May well act as a vesicular anchor by interacting with the target membrane and holding the vesicular and target membranes in proximity; Armadillo-like helical domain 
containing (962 aa)

LDHA Lactate dehydrogenase A; Belongs to the LDH/MDH superfamily. LDH family (361 aа)

Nascent polypeptide-associated complex subunit alpha, muscle-specific form; Cardiac- and muscle-specific transcription factor. May act to regulate the NACA expression of genes involved in the development of myotubes. Plays a critical role in ventricular cardiomyocyte expansion and regulates postnatal skeletal muscle growth and regeneration. Involved in the organized assembly of thick and thin filaments of myofibril sarcomeres (By similarity) (925 aa)

Epoxide hydrolase 1; Biotransformation enzyme that catalyzes the hydrolysis of EPHX1 arene and aliphatic epoxides to less reactive and more water soluble dihydrodiols by the trans addition of water (By similarity). May play a role in the metabolism of endogenous lipids such as epoxide-containing fatty acids (455 aa) 


\section{Figure 1 Kv1.3 expression and survival in human pancreatic cancer}

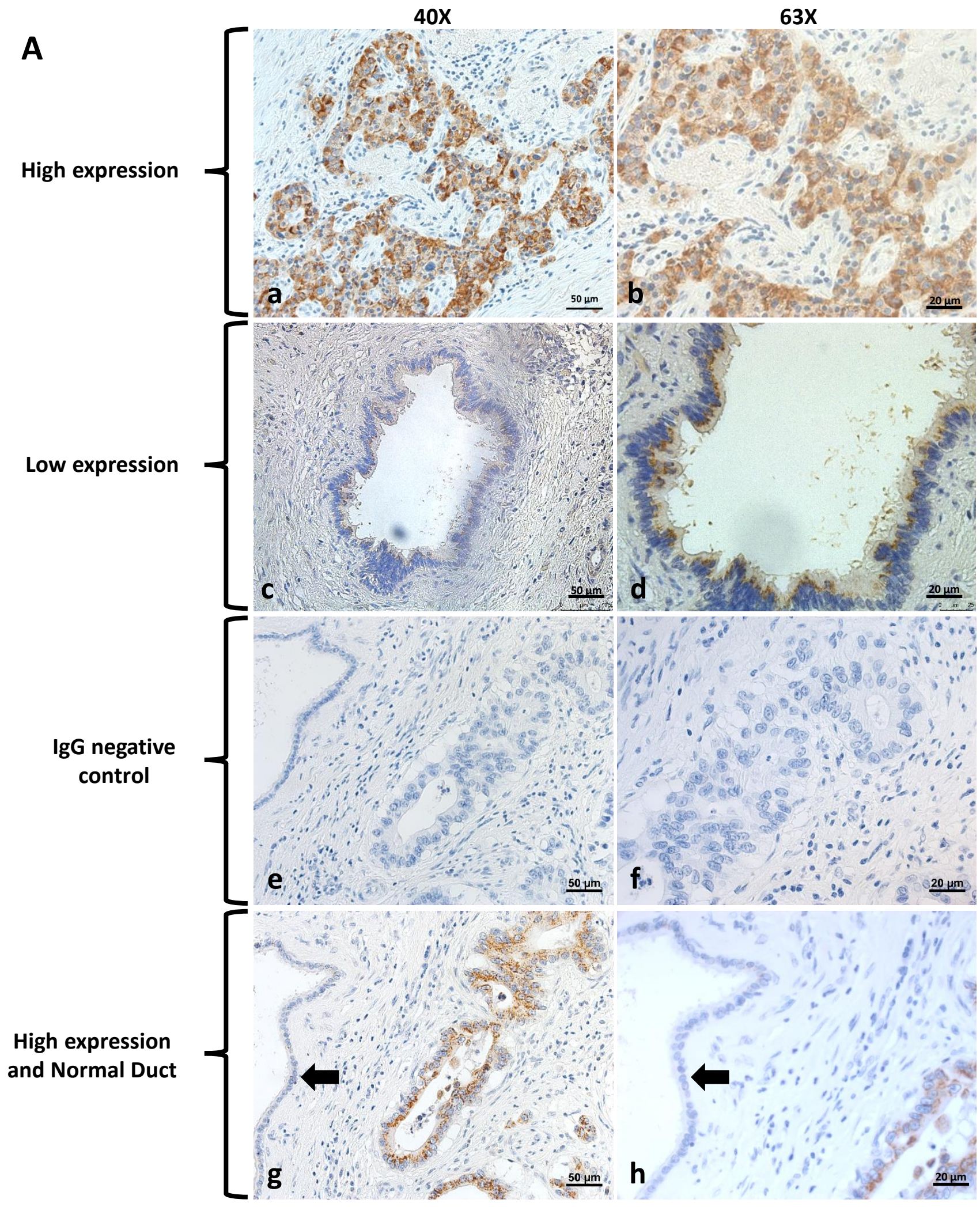


B

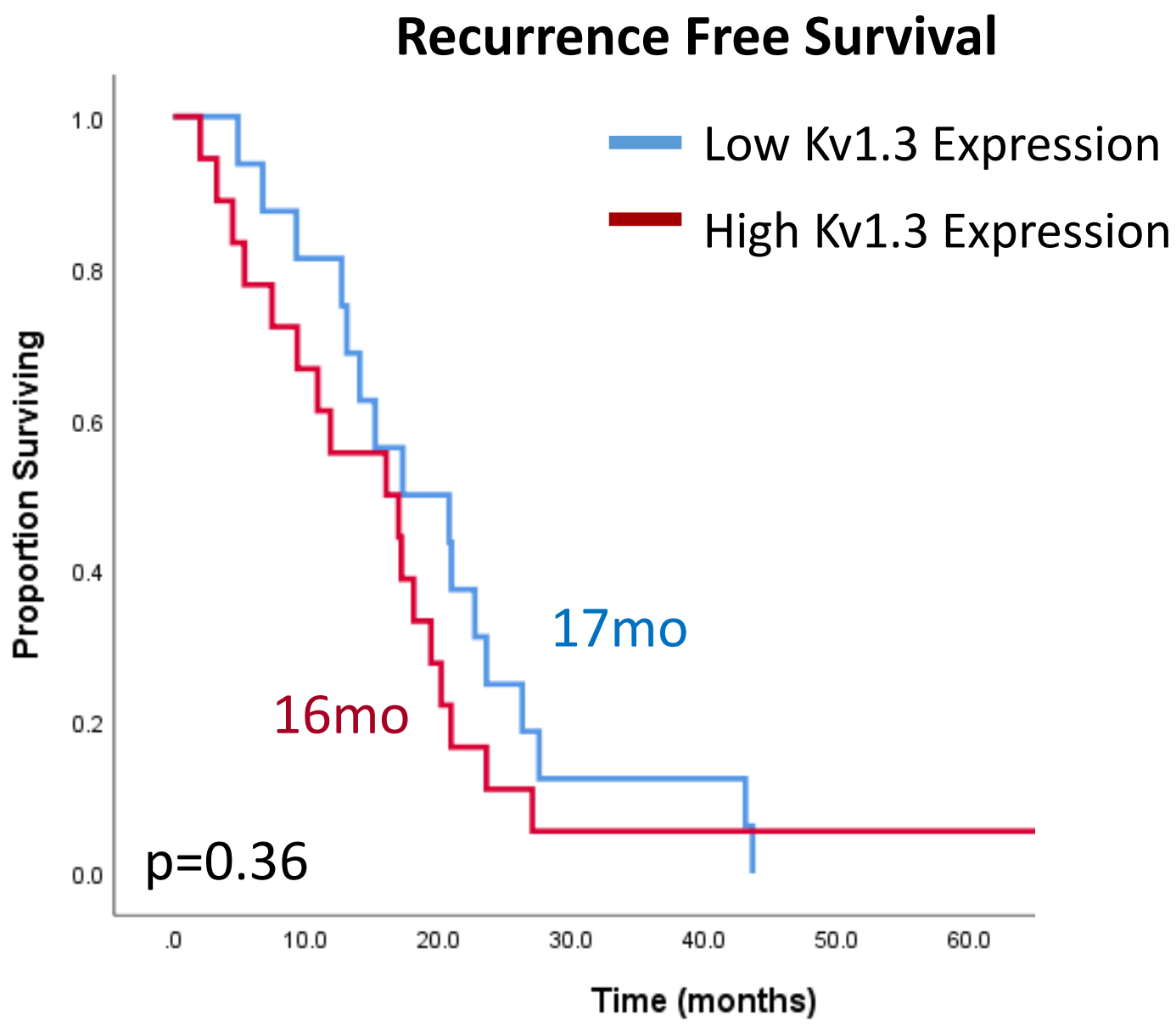

C

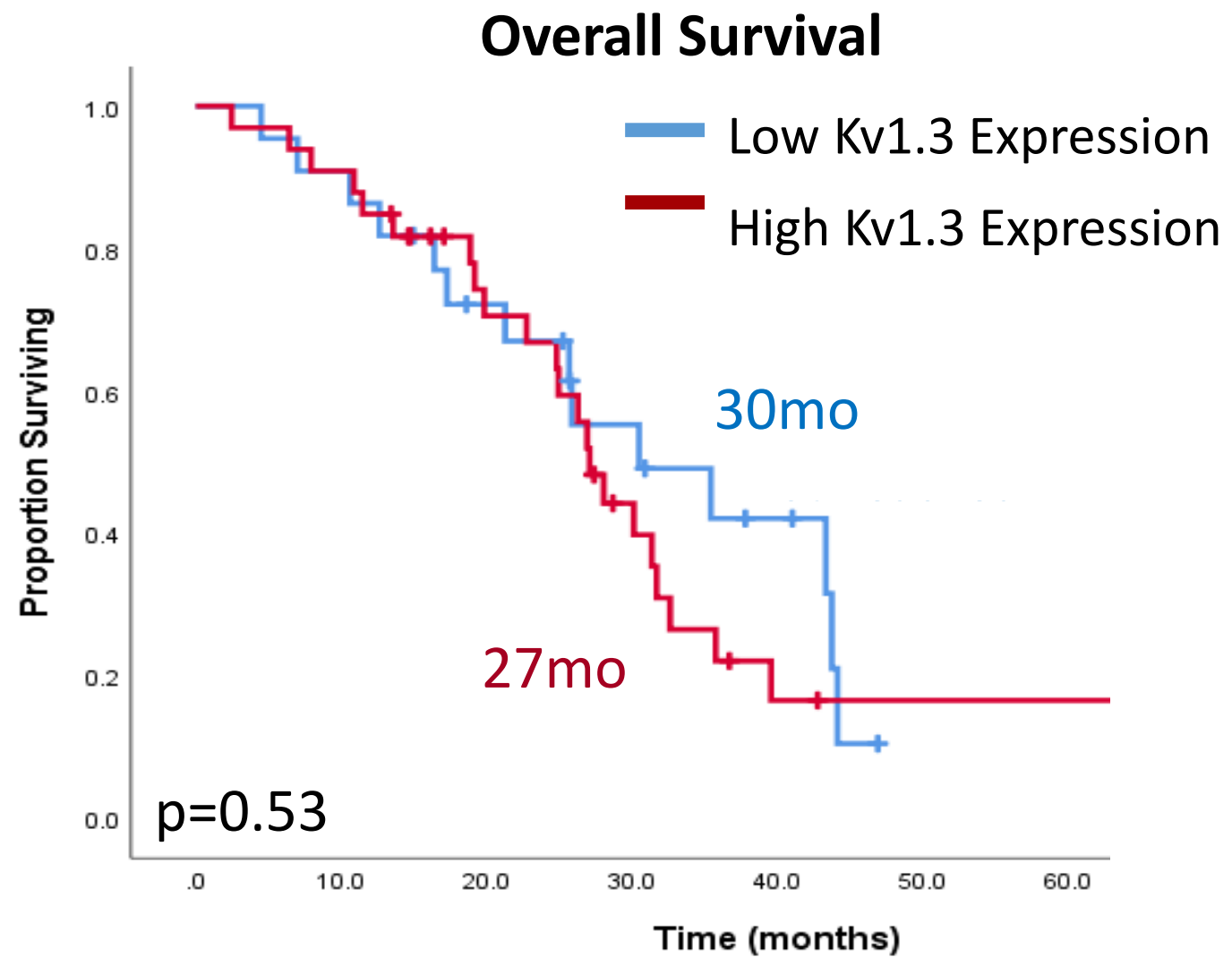


Figure 2 Effect of PCARBTP, Administered through Mini-pump, on Pancreatic Tumor Growth in an Orthotopic Mouse Model

A
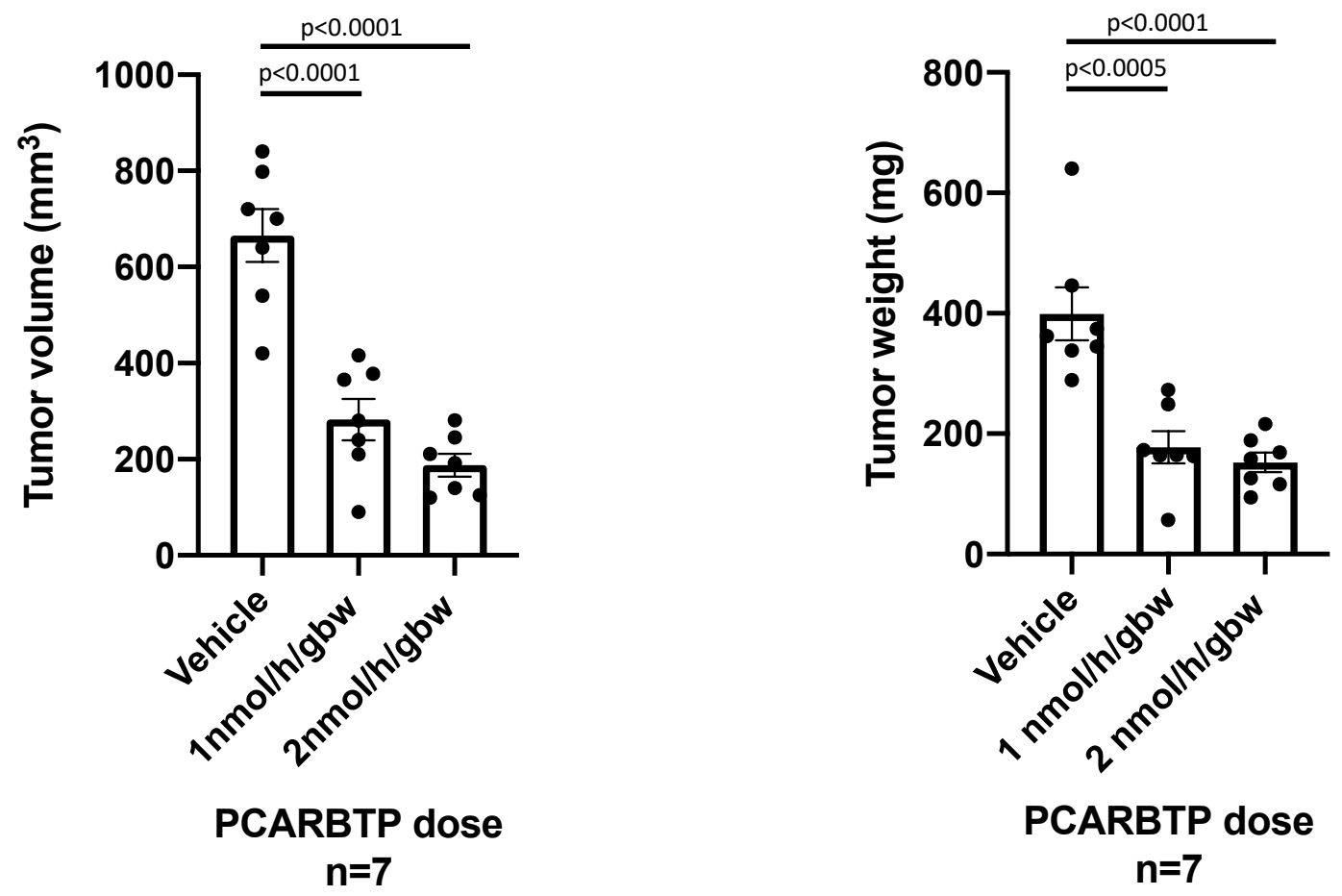

B

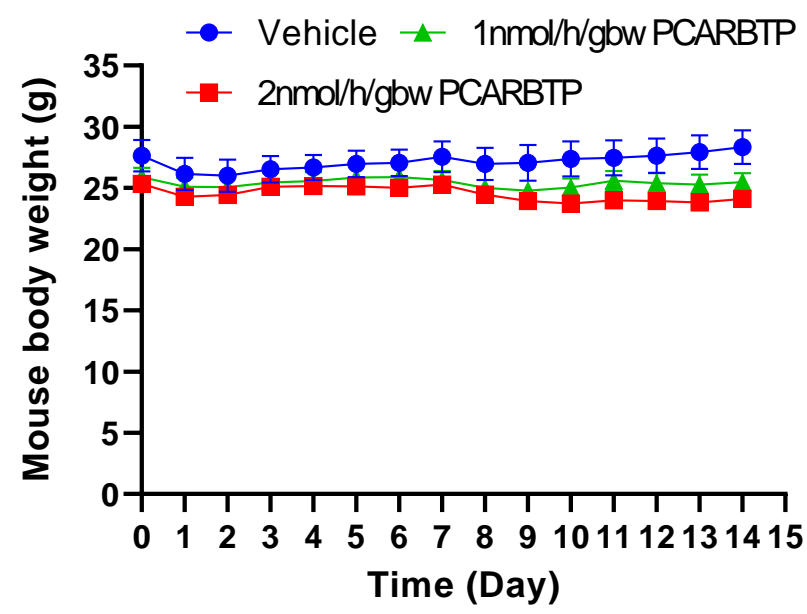

C
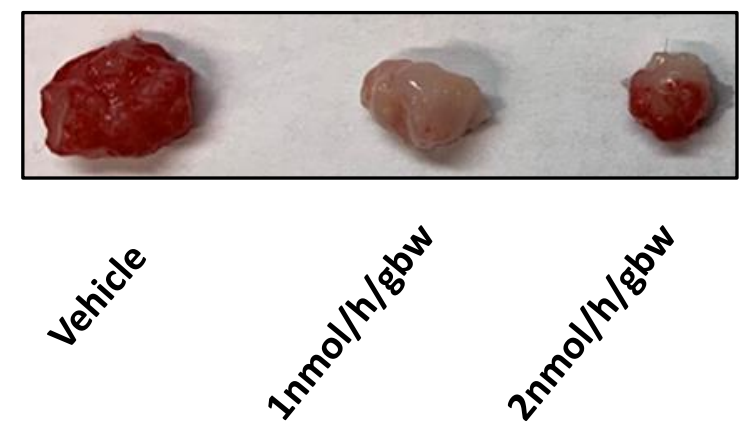

Table 1. Treatment Regimen of Figure 2 A-D

\begin{tabular}{|c|c|c|}
\hline Day 0 & Day 7-12 & Day 14 \\
\hline Before tumor injection & 1003D mini-pump duration & Tumor collected \\
\hline
\end{tabular}


Figure 3 Effect of PCARBTP, Administered Intraperitoneally, on Pancreatic Tumor Growth in an Orthotopic Mouse Model

A

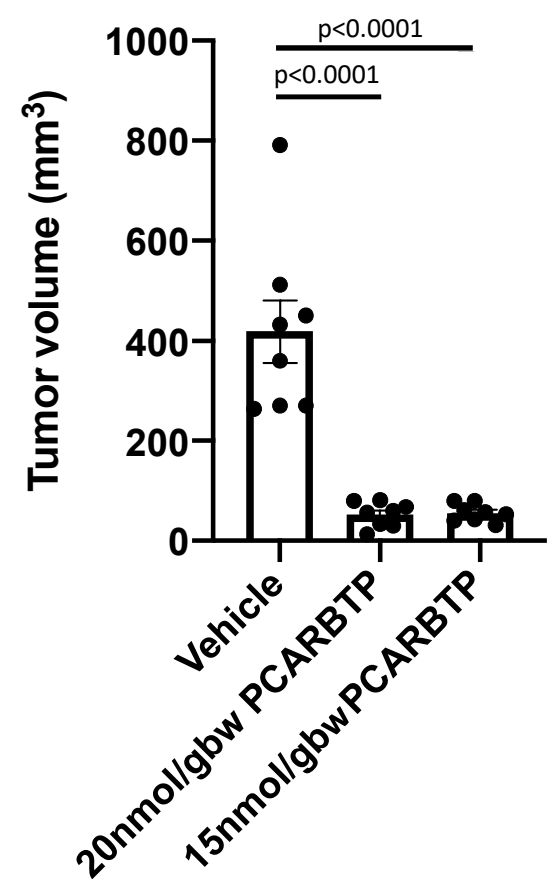

PCARBTP dose

$$
\mathrm{n}=8
$$

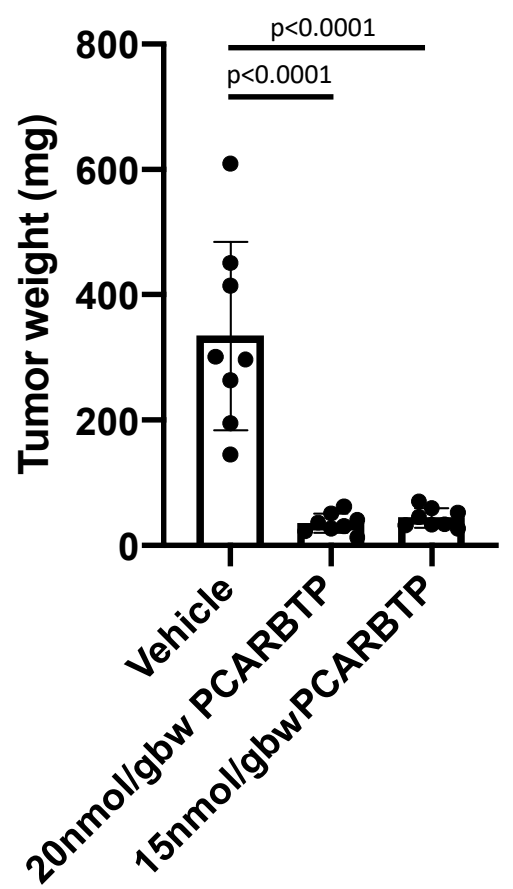

B

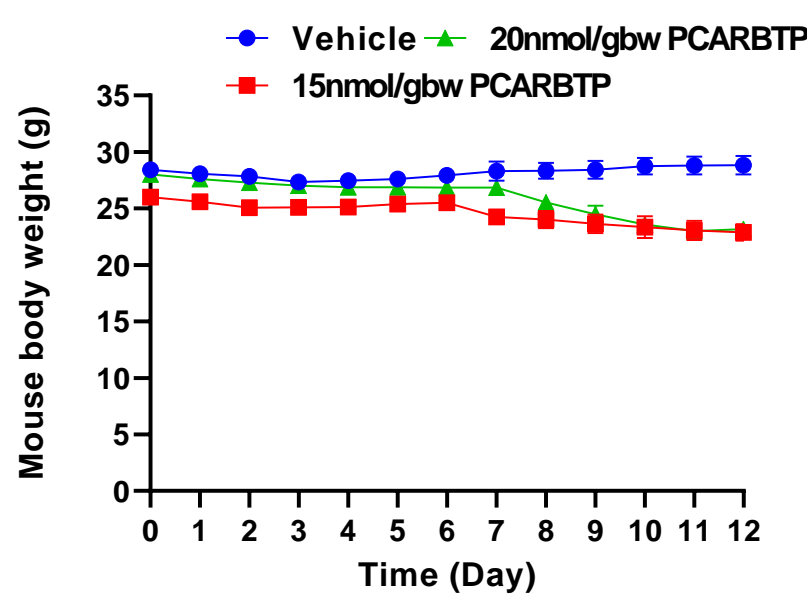

C
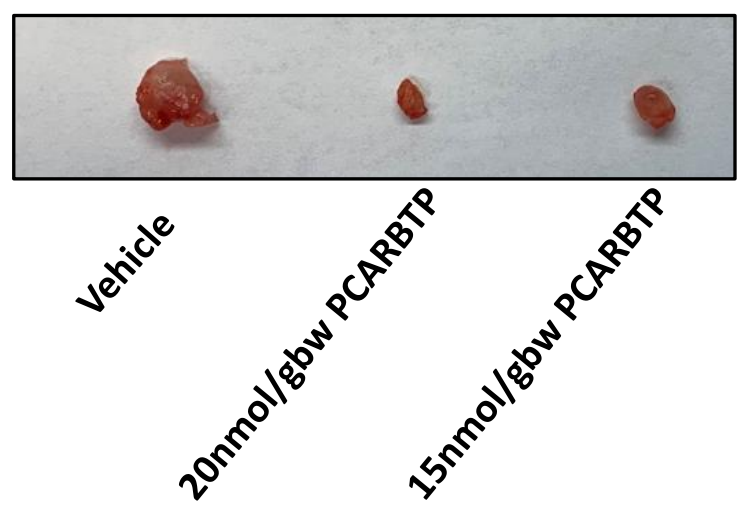

Table 2. Treatment Regimen of Figure 2 E-H

\begin{tabular}{|c|c|c|}
\hline Day 0 & Day 6, 8 and 10 & Day 12 \\
\hline Before tumor injection & 1 injection/day & Tumor collected \\
\hline
\end{tabular}


Figure 4 Effect of PAPTP, Administered Intraperitoneally, on Pancreatic Tumor Growth in an Orthotopic Mouse Model

A
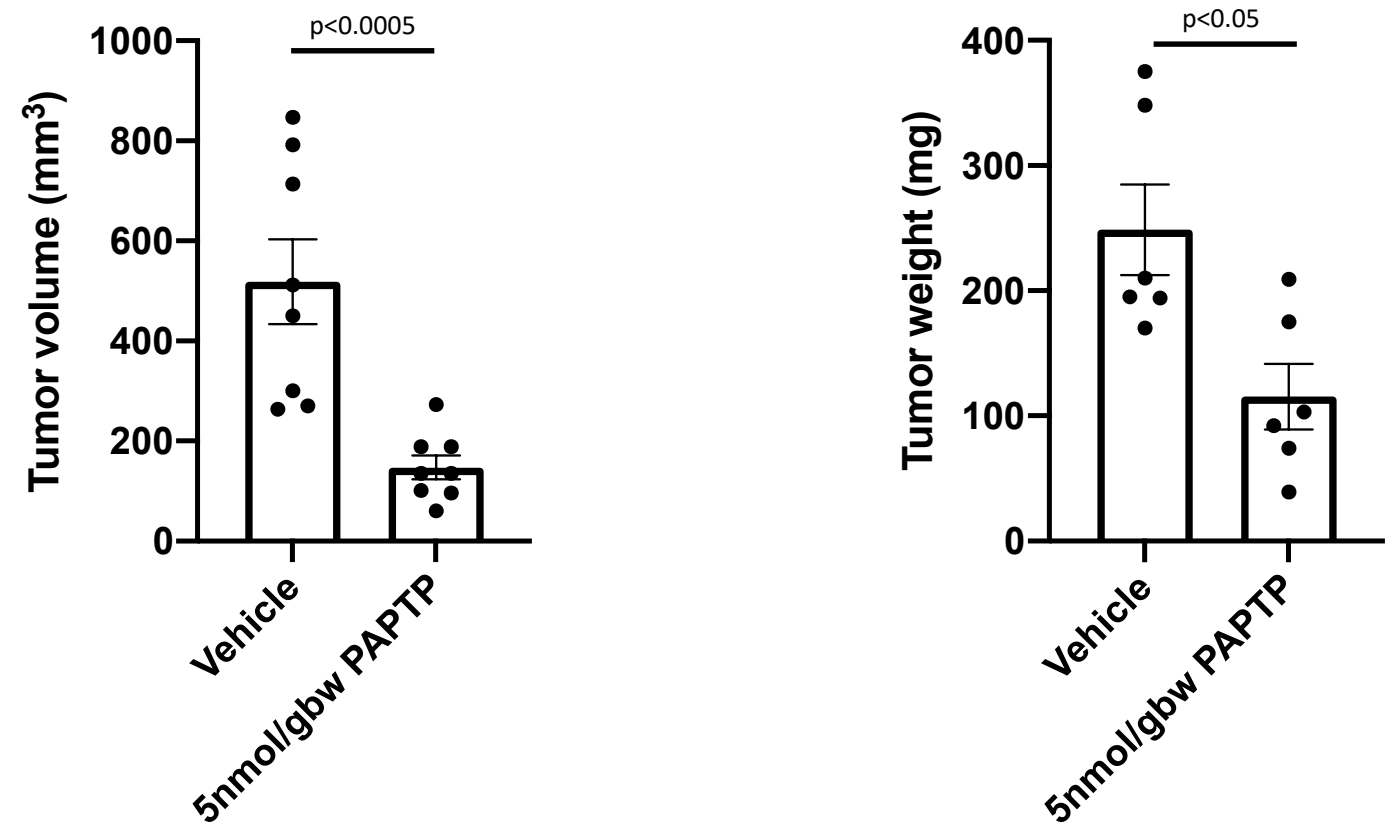

PAPTP dose $n=8$

PAPTP dose $n=8$

B

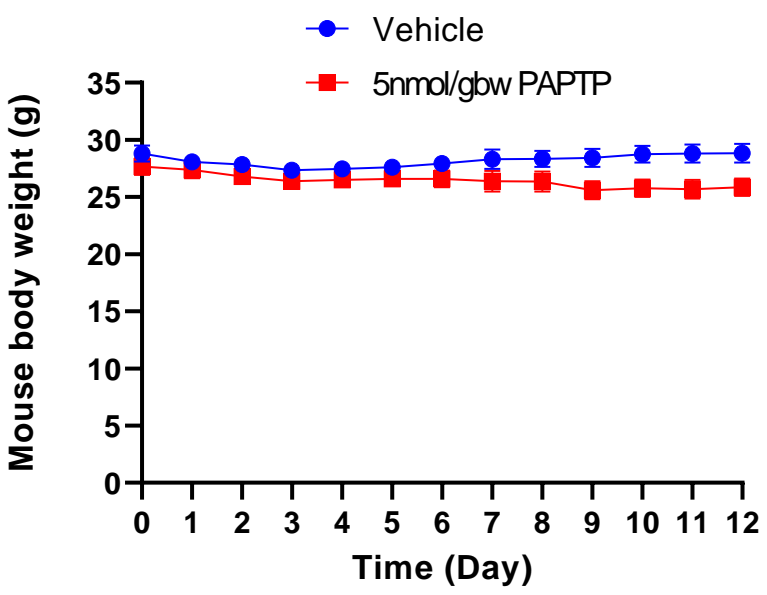

C

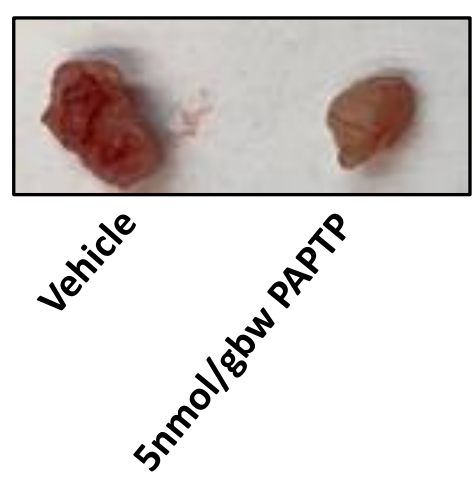

Table 3. Treatment Regimen of Figure $2 \mathrm{I}-\mathrm{L}$

\begin{tabular}{|c|c|c|}
\hline Day 0 & Day 6, 8 and 10 & Day 12 \\
\hline Before tumor injection & 1 injection/day & Tumor collected \\
\hline
\end{tabular}


Figure 5 Effect of PCARBTP/PAPTP Combined with Gemcitabine and Abraxane on Tumor Growth in an Orthotopic Mouse Model

A

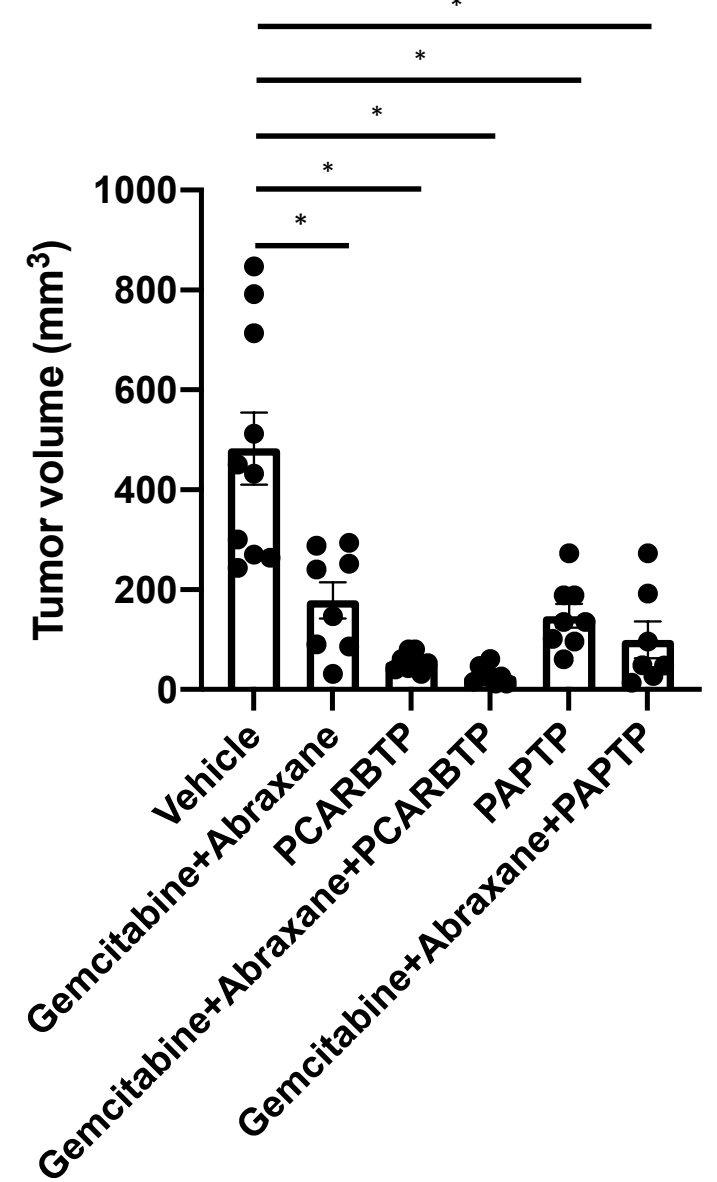

Treatment combination $n=8$
B

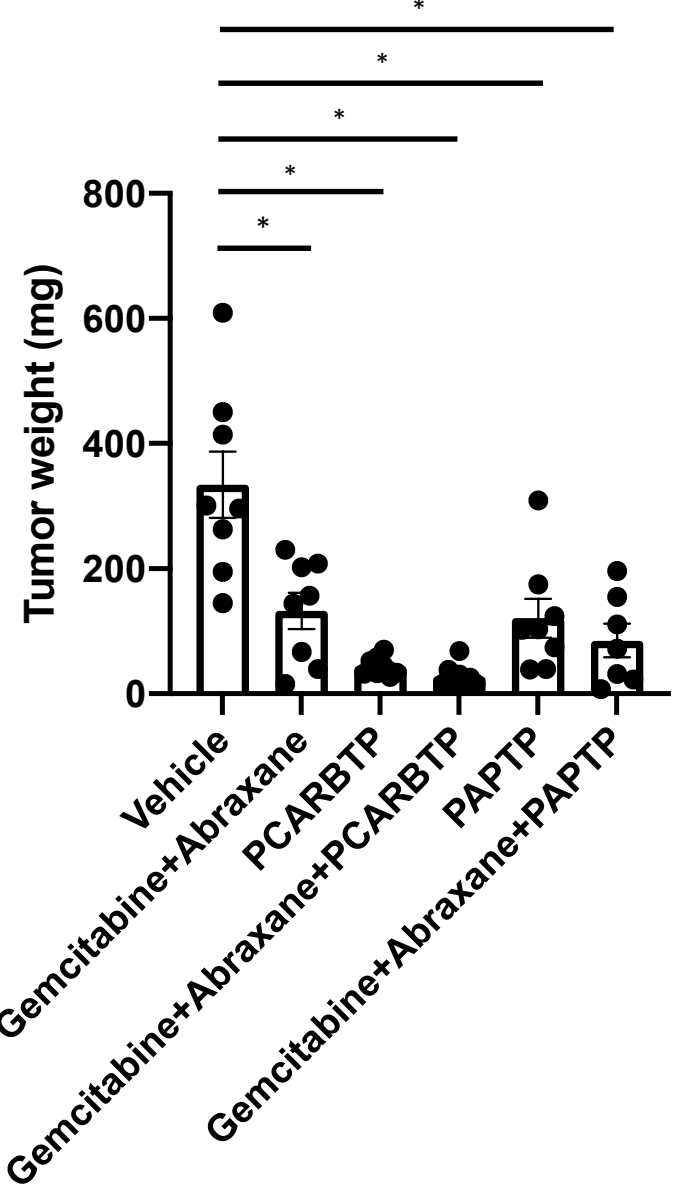

Treatment combination $n=8$

C

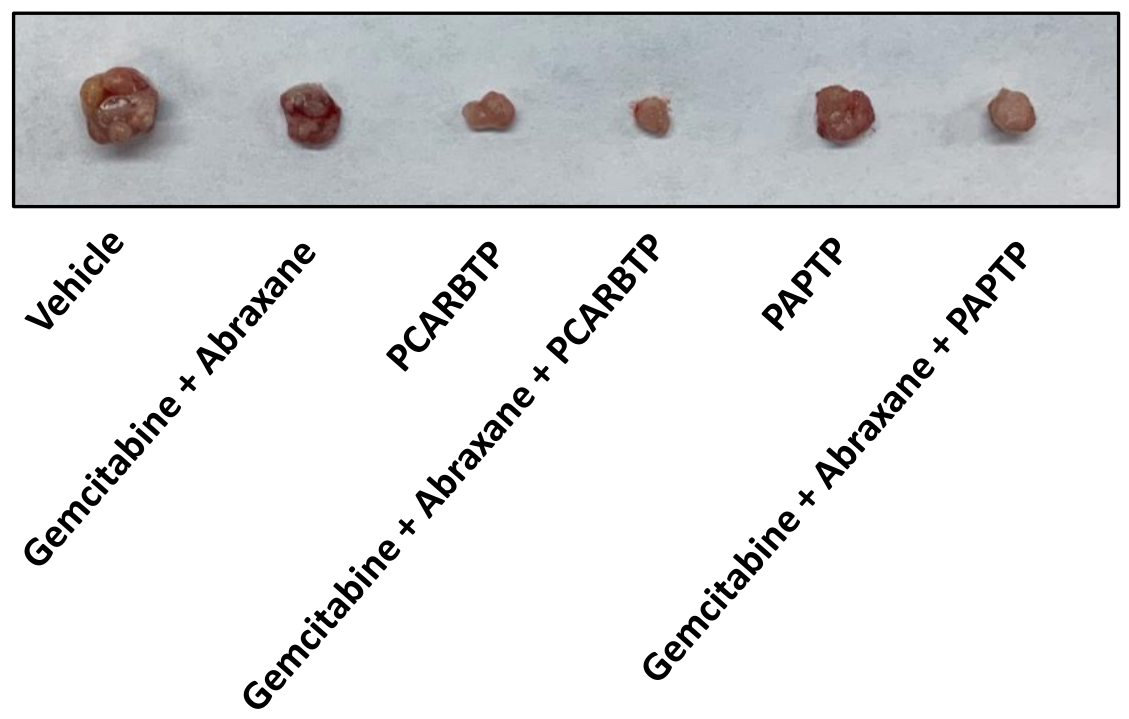

Table 4. Treatment Regimen of Figure 3

\begin{tabular}{|c|l|c|}
\hline Day 0 & Day 6, 8 and 10 & Day 12 \\
\hline Before tumor injection & 1 injection/day & Tumor collected \\
\hline
\end{tabular}


Figure 6 Tissue Toxicity After Treatment With PCARBTP/PAPTP +/Cytotoxic Chemotherapies

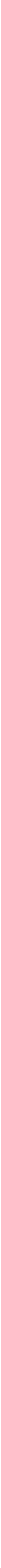


Figure 7 PCARBTP mediated cell death signal transduction pathway

A

$\begin{array}{llll}0.1 & 0.5 & 1 & 10\end{array}$

PCARBTP $(\mu \mathrm{M})$
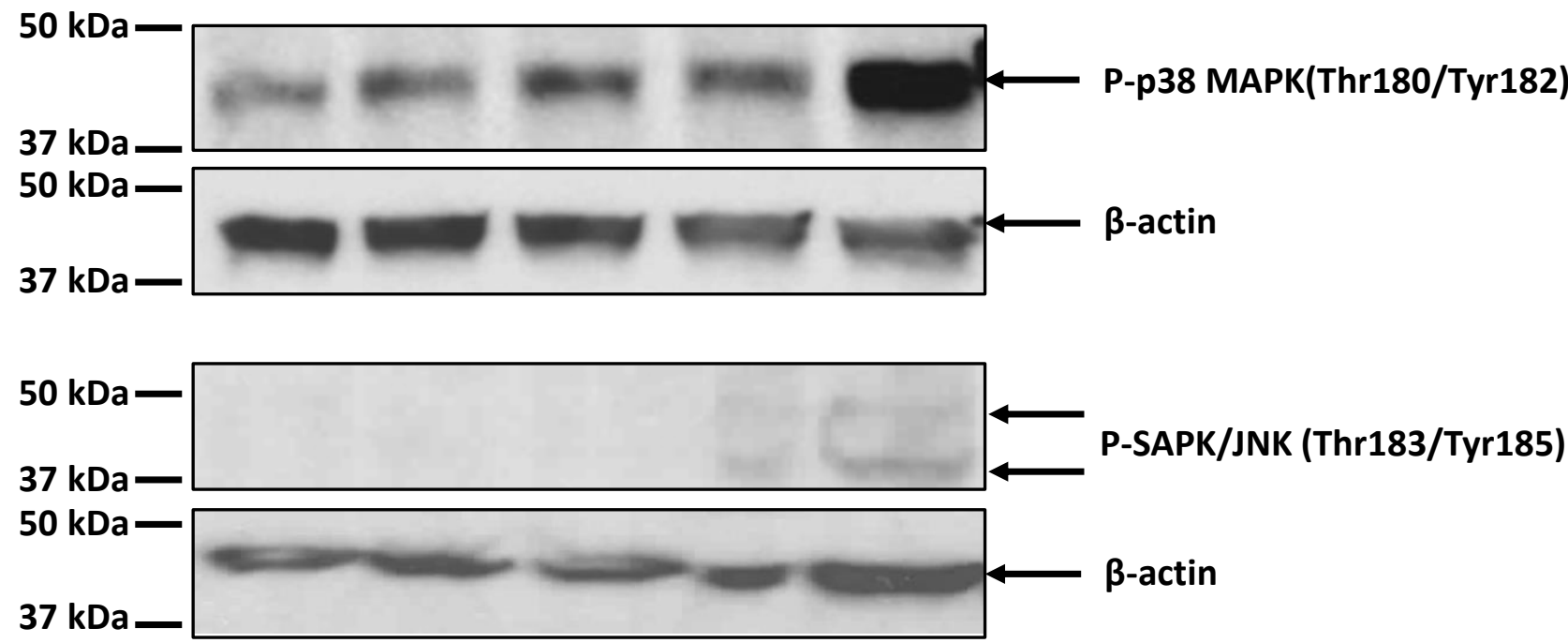

B

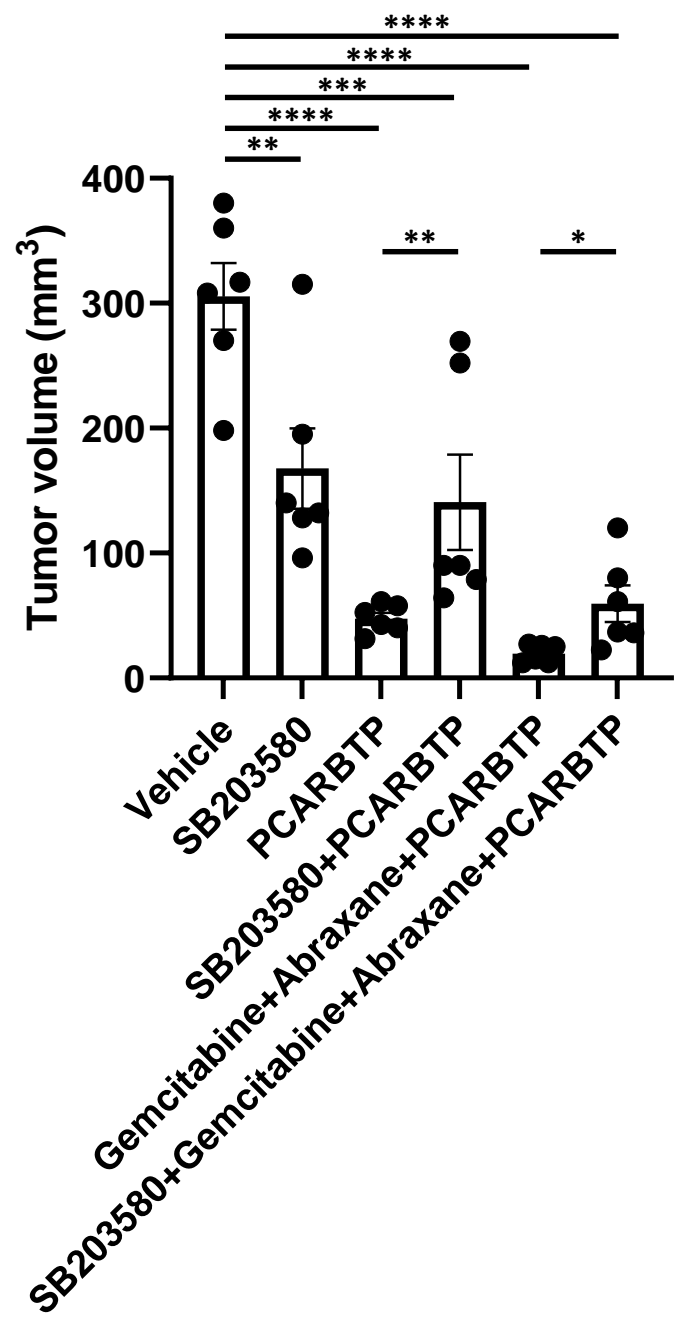

Treatment combination

$\mathrm{n}=6$

Table 5. Treatment Regimen of Figure 5 B

\begin{tabular}{|c|l|c|}
\hline Day 0 & Day 6,8 and 10 & Day 12 \\
\hline Before tumor injection & 1 injection/day & Tumor collected \\
\hline
\end{tabular}


Figure 8 Pancreatic cancer Drug resistant to PCARBTP

A

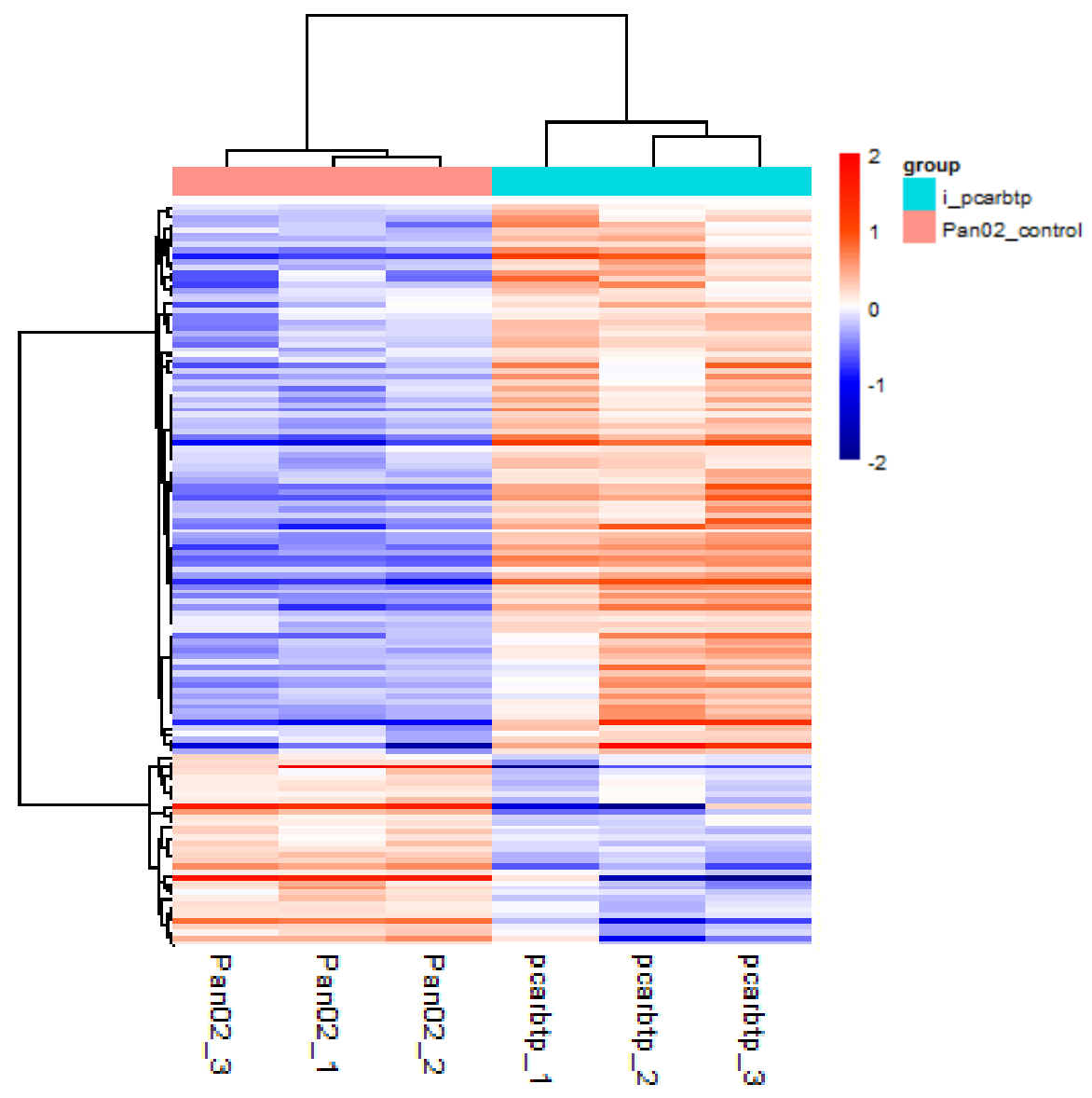

B

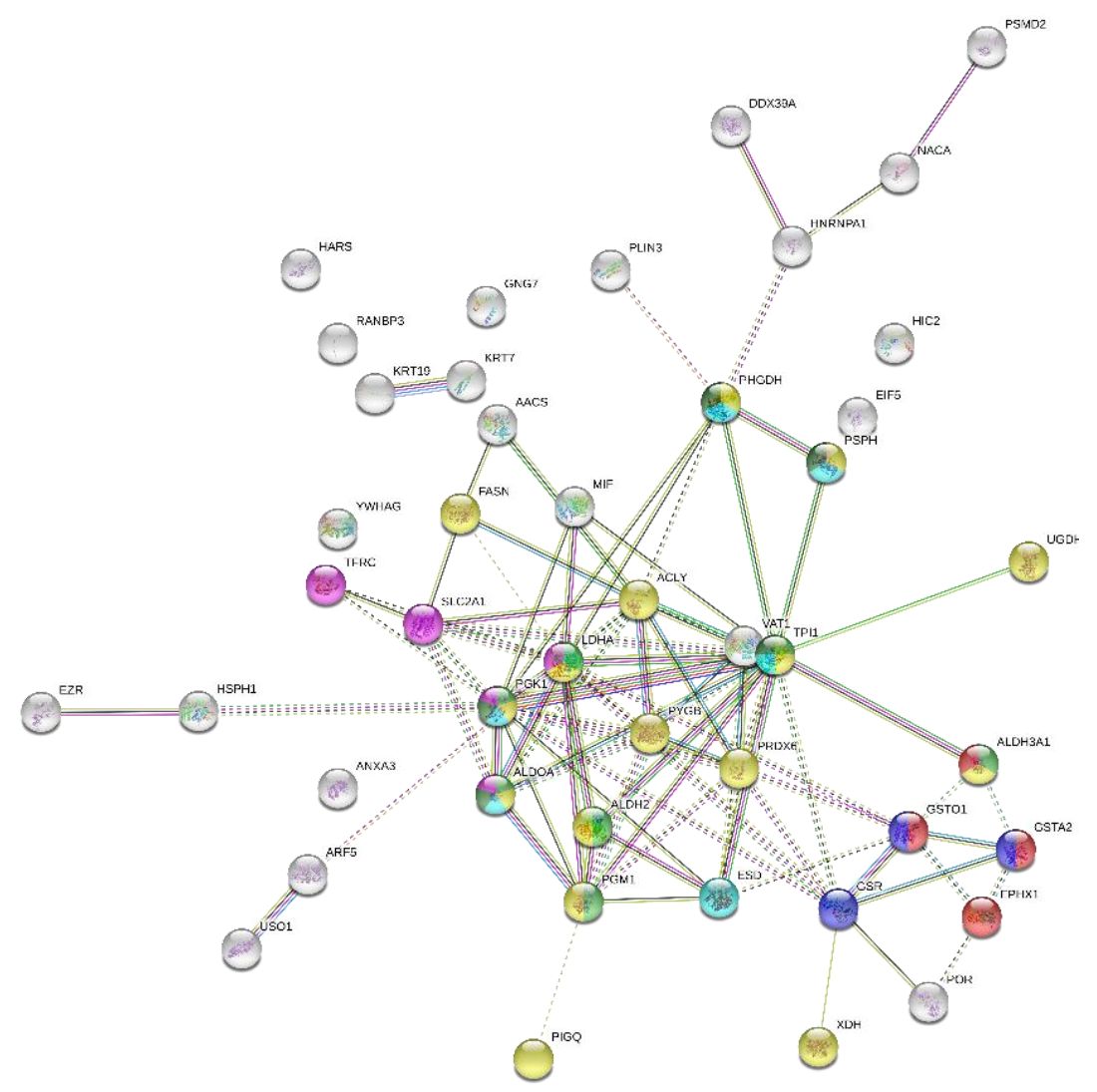

